\title{
Joint optimal lot sizing and production control policy in an unreliable and imperfect manufacturing system
}

\author{
Bassem Bouslah $^{\mathrm{a}}$, Ali Gharbi ${ }^{\mathrm{b} *}$ and Robert Pellerin ${ }^{\mathrm{a}}$ \\ ${ }^{a}$ Department of Mathematics and Industrial Engineering, École Polytechnique de Montréal, Québec, Canada; \\ ${ }^{\mathrm{b}}$ Automated Production Engineering Department, Production System Design and Control Laboratory, École de \\ technologie \\ supérieure, University of Québec, Montréal (Québec), Canada
}

\begin{abstract}
This paper deals with the problem of the joint determination of the optimal lot sizing and optimal production control policy for an unreliable and imperfect manufacturing system, where the quality control of lots produced is performed using an acceptance sampling plan. The proportion of defective items, the time between failures and the time to repair are generally distributed. The incurred total cost includes manufacturing cost, transportation cost, inspection costs, rejection cost of defective items, replacement cost for returned defective items from customers, and holding and backlog costs. The associated cost minimization problem is formulated with a stochastic dynamic programming model where the lot sizing and production rate are considered as decision variables. Given the difficulties in solving such a highly stochastic model analytically or numerically, we adopted a modified hedging point policy (HPP) to control the production rate, as well as an economic lot sizing policy for batch processing control; we also relied on a simulation-based experimental approach to determine a close approximation of the optimal control parameters. It is shown that production should be accelerated at the maximum production rate, not only when building the safety stock, as in the classical HPP, but also after rejecting a lot, in order to recuperate the loss in inventory and to maintain the on-hand safety stock. Numerical experiments and thorough sensitivity analyses are provided to illustrate the effectiveness of the proposed control policy and the robustness of the resolution approach. Some interesting behaviours regarding the impact of different parameters on the optimal decision variables are observed and discussed.
\end{abstract}

Keywords Unreliable manufacturing systems, acceptance sampling plan, optimal lot sizing, feedback control policy, simulation, response surface methodology.

\section{Introduction}

Manufacturing systems are subject to many stochastic phenomena such as random failures and repairs, imperfect production, quality deterioration, etc. Feedback control policies have proven their effectiveness in responding to such random events (Gershwin, 1994). For continuous-time failure-prone production systems, it has been shown that the well-known hedging point policy (HPP) is optimal ((Akella and Kumar, 1986), (Bielecki and Kumar, 1988)). This feedback policy controls the production rate as a function of instantaneous operational system state and inventory level, which consists in building and maintaining a safety stock during periods of system availability in order to hedge against future capacity shortages brought about by failures. 
For batch manufacturing systems, the economic production quantity (EPQ) model is often used for production-inventory control, and to determine the optimal lot sizing that minimizes overall incurred costs. Research on the EPQ model has been undertaken in different contexts of reliability and/or quality imperfection, especially over the last two decades. In one of the pioneering papers that addressed the EPQ problem of unreliable batch manufacturing systems, Groenevelt et al. (1992a) investigated the impact of system breakdowns and corrective maintenance on production lot sizing decisions. Assuming a deterministic constant production rate, negligible repair time, exponential failures and no backlogs, the authors determined the optimal lot sizing for two production reorder policies (no-resumption (NR) policy and abort/resume (AR) policy). In a subsequent study, Groenevelt et al. (1992b) defined a production control policy to simultaneously determine the optimal lot sizing and the safety stock level that satisfy a prescribed service level. They assumed that during a production run, a certain fraction of the items produced is instantaneously diverted into the safety stock. Kim and Hong (1997) and Kim et al. (1997) extended the Groenevelt et al. (1992a) model, which assumes that the times between failures follow general distributions. Chung (1997) determined an approximate formula for the optimal lot sizing of the Groenevelt et al. (1992a) model by calculating its bounds. Giri et al. (2005) focused on the problem of EPQ for an unreliable production system where the production rate is treated as a decision variable. They developed two models: with and without safety stock, in order to jointly determine the optimal lot sizing and the optimal feasible production rate. Giri and Dohi (2005) extended the Giri et al. (2005) model with safety stock, taking into account preventive maintenance, and assuming that the failure and repair times are general distributions. Bouslah et al. (2011) obtained an integrated optimal lot sizing and feedback production policy, considering a transportation delay of lots produced and added to the serviceable stock. All the above-cited studies deal with the effect of process reliability on the EPQ model, and do not consider the quality issue, instead, assuming that all produced items are of perfect quality.

On the other hand, many works have considered the quality imperfection problem in the EPQ model, without reliability. Porteus (1986) and Rosenblatt and Lee (1986) were among the first researchers who investigated the effect of quality imperfection on the EPQ. In both studies, they assumed that the deterioration of production system is a random process characterized by two states: the 'in-control' state, when all items produced are of conforming quality, and the 'out-ofcontrol' state, when some percentage of items produced are defective. Lee and Rosenblatt (1987) considered the maintenance by inspection feature to monitor deterioration of the production process: if inspections indicate that the production process is 'out of control', it will be restored to the 'in-control' state. They then focused on simultaneously determining the EPQ and optimal inspection schedules. Khouja and Mehrez (1994) formulated an EPQ model assuming that production rate is a decision variable and that the quality of the production process deteriorates with an increased production rate. Salameh and Jaber (2000) presented a modified inventory model which extends the traditional EPQ model by accounting for imperfect quality items. Hayek and Salameh (2001) derived an optimal operating policy for an EPQ model under the effect of imperfect quality. They assumed that all defective items produced are reworked and added to 
perfect quality inventory, and that shortages are allowed and backordered. Ben-Daya (2002) developed an integrated model for the joint determination of the EPQ and preventive maintenance level for an imperfect process having a general deterioration distribution with an increasing hazard rate. Chiu (2003) extended the Hayek and Salameh (2001) model, by assuming that not all of the defective items produced are reworked, and that a portion of all imperfect quality items are scrapped and discarded before the rework process is started. Finally, Sana (2010) considered that the percentage of defective items varies linearly with both the production rate and the productionrun time, and that the probability distribution of the shift time from the 'in-control' to the 'out-ofcontrol' state also depends on the production rate. Therefore, he focused on determining the optimal lot sizing and the optimal production rate.

In the aforementioned EPQ models, reliability and quality issues are studied separately. However, these two problems are often observed simultaneously in real-life manufacturing systems. Only few recent EPQ models jointly consider the effects of equipment breakdowns and quality deterioration in the production process. Among these works, Chiu et al. (2007) extended the works of (Chung, 1997) and (Chiu, 2003) in order to determine the optimal run time problem of EPQ models with scrap, reworking of defective items, and stochastic machine breakdowns. Liao et al. (2009) integrated maintenance programs (perfect/imperfect preventive maintenance and imperfect repair) with the EPQ model for an imperfect and unreliable manufacturing system. Chakraborty et al. (2009) developed integrated production, inventory and maintenance models in order to study the joint effects of process deterioration, machine breakdown and inspections on optimal lot sizing decisions. Sana and Chaudhuri (2010) extended the Giri and Dohi (2005) model, considering the effect of an imperfect production process subject to random breakdowns and variable safety stocks. The proposed policy consists in determining the optimal safety stock, the optimal production rate and the optimal lot sizing.

In most existing EPQ models, the effects of using such a quality control policy on the production policy parameters (including lot sizing) have not been sufficiently studied. Indeed, inspection is considered only as a tool for controlling the quality deterioration of the production process. As well, most models assume (except for a few works like Ben-Daya (2002)), that the inspection delay is negligible. However, inspection is in itself an important part of quality assurance, which should be fairly represented in the EPQ model. Some authors, such as Salameh and Jaber (2000), assume that all lots produced are 100\% inspected. Liao et al. (2009) consider a complete quality audit using automated inspection. From an economic point of view, the cost of a $100 \%$ inspection is very high, particularly with automation systems requiring high technology. According to Chin and Harlow (1982), the inspection process is normally the largest single cost in manufacturing.

In manufacturing organisations, statistical techniques, such as control charts and acceptance sampling plans, can be used for quality control when the cost of a $100 \%$ inspection is higher than the cost of delivering a certain proportion of defective items (Besterfield, 2009). Only few researchers have integrated quality control techniques into EPQ models such as Ben-Daya (1999) who presented an integrated model for the joint optimization of production quantity, design of quality control parameters using the $\bar{x}$-control chart and the maintenance level. 
Another common assumption made in most EPQ models is that the lot which is currently being processed can instantly meet the demand, and even build a safety stock if the production rate is strictly greater than the demand rate. This assumption is unrealistic for a wide range of manufacturing systems where a certain delay, for lot sampling, inspection, reworking, etc., exists between the production and the final stock that truly serves the demand.

Considering the limitations of past models in the context of quality and production-inventory control, the main purpose of this paper is to develop an integrated optimal lot sizing model and a feedback control policy for unreliable and imperfect batch manufacturing systems, where quality control is performed using a single acceptance sampling plan. The problem is formulated using a stochastic dynamic programming in which the lot sizing and the production rate are considered as decision variables. Since it is difficult to analytically or numerically derive the optimal solution of the model, we adopt a modified HPP to control the production rate and a feedback-inventory to control the batch processing order using theoretical arguments and approximations. Then, we use a simulation-based experimental approach to determine the optimal parameters of the proposed control policy, when the proportion of defective items, and failure and repair times follow general distributions.

The remainder of this study is organized as follows. Section 2 introduces the notation and the problem description, and presents a formulation of the optimal production problem. Section 3 presents the proposed control policy. Section 4 describes the resolution approach used to determine the optimal design of the control policy and the optimal incurred cost. Section 5 provides an illustrative numerical example of the resolution approach. A thorough sensitivity analysis is given in section 6 , in order to confirm the robustness of the resolution approach and to study the behaviour of the system under different settings of cost and quality parameters. Finally, section 7 concludes this paper.

\section{Problem formulation}

\subsection{Notation}

The following notation will be used throughout the paper:

$\begin{array}{ll}q(t) & \text { WIP lot level at time } t \text { (units) } \\ x(\mathrm{t}) & \text { Inventory level at time } t \\ y(\mathrm{t}) & \text { Inventory position at time } t \\ u(.) & \text { Production rate (units/time) } \\ u^{i} & \text { Production rate of the } i \text { th lot (units/time) } \\ u_{\max } & \text { Maximum production rate (units/time) } \\ d & \text { Constant demand rate (units/time) } \\ \Phi(p) & \text { Probability density function associated to the proportion of defective items } \\ p_{i} & \text { Proportion of defective items in the } i \text { th lot } \\ \bar{p} & \text { Long-term average proportion of defective items in a lot } \\ n & \text { Sample size } \\ c & \text { Acceptance number }\end{array}$




$\begin{array}{ll}P_{a} & \text { Average probability of acceptance } \\ Q & \text { Lot sizing (units) } \\ \theta_{i} & \text { Production start time of the } i \text { th lot } \\ \delta_{i} & \text { Production end time of the } i \text { th lot } \\ Q^{i} & \left.\text { ith lot produced during the interval }] \theta_{i}, \delta_{i}\right] \\ N(t) & \text { Cumulative total number of lots produced and inspected at time } t \\ \sim T B F & \text { Probability density function associated to the Time Between Failures } \\ \sim T T R & \text { Probability density function associated to the Time To Repair } \\ \tau_{\text {insp }} & \text { Inspection delay (time) } \\ C_{\text {hold }} & \text { Unit holding cost (\$/unit) } \\ C_{\text {back }} & \text { Unit backlog cost (\$/unit) } \\ C_{p r o d} & \text { Unit production cost (\$/unit/time) } \\ C_{t r} & \text { Cost of transportation of a lot (\$/load) } \\ C_{\text {insp }} & \text { Unit inspection cost (\$/unit) } \\ C_{r e j} & \text { Unit rejection cost (\$/unit) } \\ C_{\text {rep }} & \text { Unit replacement cost (\$/unit) }\end{array}$

\subsection{Problem description \& assumptions}

The manufacturing system under study consists of an unreliable production facility supplying a downstream stock (Figure 1). One single item is manufactured in lots in order to meet a constant and continuous demand. The work-in-process (WIP) lot is stored in a downstream area of the facility until the production lot is completed. We assume that when a failure occurs during the production cycle, the production of interrupted lots is always resumed after repair.

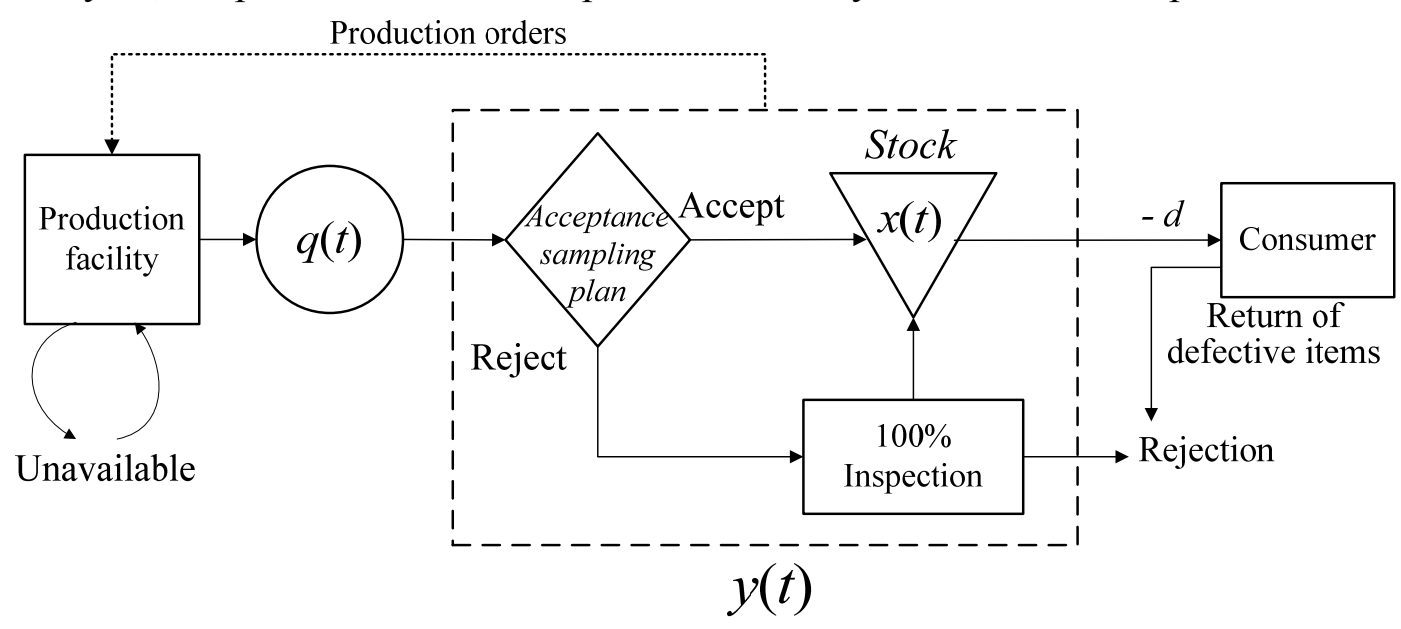

Figure 1. Unreliable and imperfect manufacturing system with quality control.

As the production quality is imperfect, a certain proportion of defectives occur during the production process. In this study, we assume that discovered defective items are scrapped. This is often observed in the chemical, food and printing industries (Min and Galle, 1997). As a lot-to-lot variation exists in manufacturing, we assume that the proportion of defective items also varies 
from lot-to-lot following a prior known distribution with probability density function $\Phi(p)$. Several forms of the prior distribution of $\Phi(p)$ has been proposed by Hald (1960) and Chiu (1974). Let $p_{1}, p_{2}, . ., p_{\infty}$ be respectively the discrete proportion of defective items in the $Q^{I}, Q^{2}, \ldots$, $Q^{\infty}$ lots generated from the known probability function $\Phi(p)$. As in Salameh and Jaber (2000) and Kahn et al. (2011), we assume that the number of defective items in each $i$ th lot is equal to $p_{i} Q$, proportionally to the lot sizing $Q$. Let $X$ be the random variable denoting the number of defective items in the lot $Q$. We assume that the production process is under binomial control. Therefore, the probability density function $f_{Q}(X)$ of the number of lot defectives $X$ is given by

$$
f_{Q}(X)=\int_{0}^{1} C_{k}^{n} p^{X}(1-p)^{Q-X} \Phi(p) \cdot d p
$$

where $p$ denotes the proportion of defective items.

Once produced, the lot is transported to the inspection area where a quality control is performed on the lot to decide whether or not it is acceptable. The quality control policy consists of a lot-bylot acceptance sampling plan with parameters $n$ and $c$. A sample of size $n$ is drawn randomly from the lot, and inspected item-by-item by attributes. Let $Y$ be the random variable denoting the number of defective items in the sample of size $n . Y$ is described by the conditional distribution $p\{Y \mid X\}$ such that the simultaneous distribution of $X$ defectives items in the lot and $Y$ defectives items in the sample is given by

$$
p\{X, Y\}=f_{Q}(X) p\{Y \mid X\}
$$

and the marginal distribution of $Y$ which represents the average probability of $Y$ defectives items in the sample $n$ is given by

$$
m_{n}(Y)=\sum_{X} p\{X, Y\}=\sum_{X} f_{Q}(X) p\{Y \mid X\}
$$

Hald (1960) developed a theory to fit $m_{n}(X)$ by a well-known compound hypergeometric distribution which is combination of the prior distribution $f_{Q}(X)$ and the conditional hypergeometric distribution $p\{Y \mid X\}$. The Hald's theory has been used later by Peters et al. (1988), Ben-Daya et al. (2006) and Ben-Daya \& Noman (2008) in order to integrate the quality control using acceptance sampling plan with the Economic Order Quantity model.

The sample inspection duration is equal to $n \tau_{\text {insp }}$. If the number of defective items $Y$ in the sample does not exceed the acceptance number $c$, the lot is accepted and the $Y$ defective items are replaced from a stock of known good items before the transport of the entire lot to the final stock area. As the acceptance number $c$ is generally a very small number comparing with the sample size $n$, we consider that the time of replacement of $Y$ defective items is negligible. Otherwise, the lot is rejected. In this case, a $100 \%$ inspection is performed on the lot and all defective items are 
sorted by inspection personnel. The duration of this operation is equal to $(Q-n) \tau_{\text {insp }}$. For each $100 \%$ inspection of a non-accepted $i$ th lot, there are $p_{i}(Q-n)$ defective items to be discarded and rejected. Only the $\left(1-p_{i}\right) Q$ good items will be transported to the final stock. Let $\xi_{i}$ be the arrival time of the $i$ th lot to the on-hand serviceable inventory $x($.$) . Then, \xi_{i}=\delta_{i}+n \tau_{\text {insp }}$, if the $i$ th lot is accepted, and $\xi_{i}=\delta_{i}+Q \tau_{\text {insp }}$, if not. We assume, in our study, that $\xi_{i} \leq \delta_{i+1}(i=1 . . N)$, which means that the quality control operations of the $i$ th lot is finished before the end of production of the next $i+1$ th lot. Figure 2 depicts the dynamic of production (WIP lot level), and the evolution of the serviceable inventory level as a function of instantaneous system availability, production cycle length, and acceptance or rejection of lots produced.

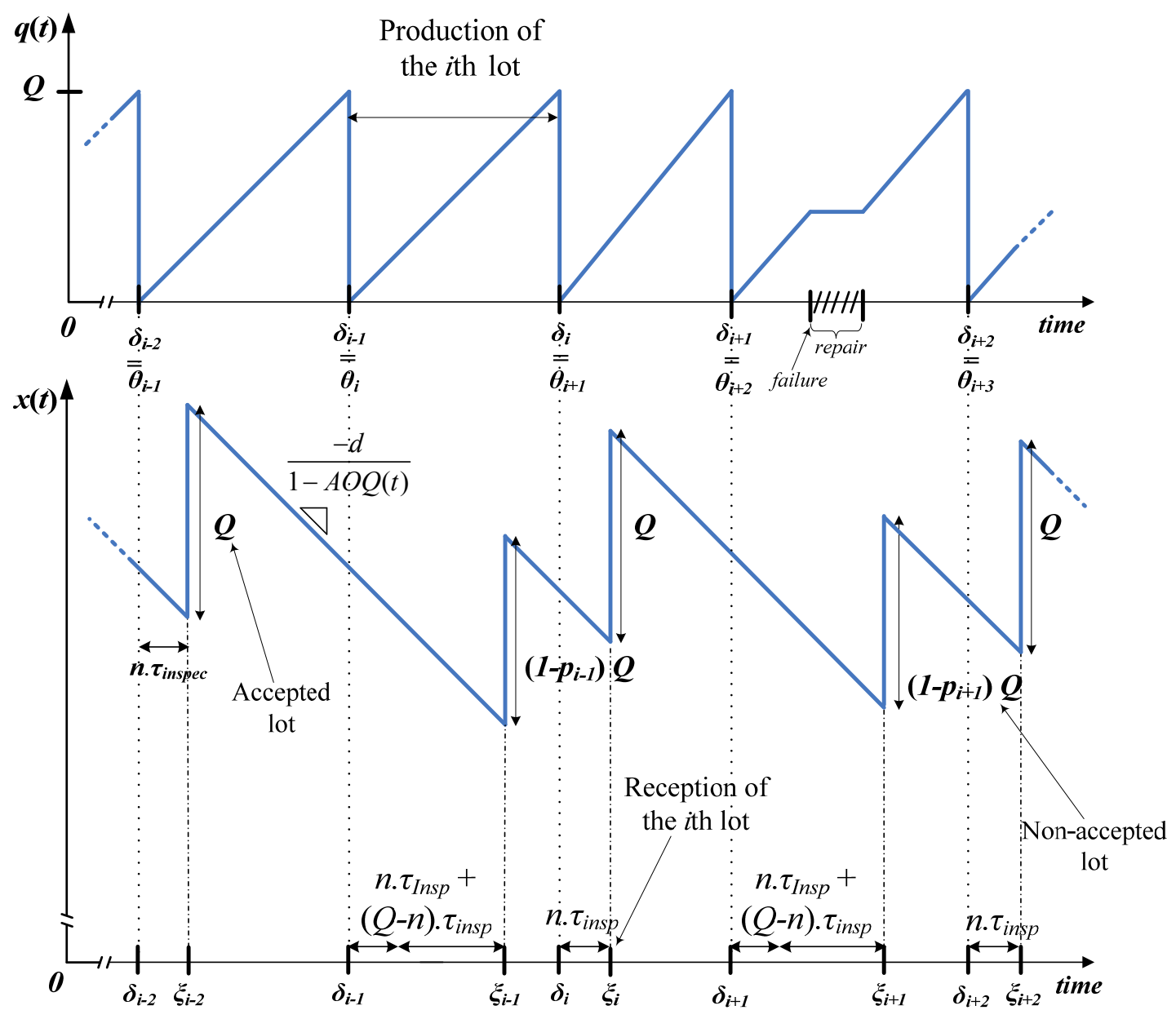

Figure 2. Production and inventory level dynamics.

The probability $P_{a}\left(p_{i}\right)$ of accepting the $i$ th lot for given proportion of defective items $p_{i}$ can be calculated using the binomial probability distribution (Besterfield, 2009), as follows:

$$
P_{a}\left(p_{i}\right)=\sum_{k=0}^{c} C_{k}^{n}\left(p_{i}\right)^{k}\left(1-p_{i}\right)^{n-k}
$$


As the proportion of defective items varies from lot-to-lot, the probability of acceptance of lots produced varies also from lot-to-lot. From Eq.(3), the average probability of acceptance $P_{a}$ of the lots produced is given by

$$
P_{a}=\sum_{Y=0}^{c} m_{n}(Y)=\sum_{Y=0}^{c} \sum_{X} p\{X, Y\}
$$

As the accepted lots do not undergo 100\% inspection, the defective items existing in these lots will reach the final stock, and will therefore be transmitted to the consumer. The average proportion of defective items that contain the final stock, also named the Average Outgoing Quality $A O Q$, can be calculated instantaneously using the following formula:

$$
A O Q(t)=\frac{\sum_{i=1 / a^{i}=1}^{N(t)} p_{i}(Q-n)}{\sum_{i=1}^{N(t)} a^{i} Q}
$$

Where, $a^{i}=\left\{\begin{array}{ll}1 & \text { if the } i \text { th lot is accepted } \\ 1-p_{i} & \text { if the } i \text { th lot is rejected }\end{array}\right.$.

In order to study the effect of outgoing quality on the control policy sought, we assume that, in the producer-consumer relationship, all defectives items are returned to the producer and replaced by good ones. While the demand/backlog is filled, the returned quantity at each time $t$ is considered proportional to the demand rate $d$, and is replaced by good items immediately. Then, the real demand rate when the demand is served becomes equal to $d /(1-A O Q(t))$.

Our objective is to determine the optimal lot sizing and the optimal control policy that minimize the total expected cost, including inventory cost related to holding WIP and finished products, shortage cost related to the backlogged demand, production cost depending on the production rate value, inspection cost for each lot sampling, replacement cost for returned defective items, 100\% inspection and rejection costs for each non-accepted lot, and transportation cost for each lot produced.

\subsection{Optimization problem formulation}

The manufacturing system behaviour changes randomly with time. It can be characterized at each time $t$ with four continuous-time components including:

- A stochastic process $\{\alpha(\mathrm{t})\}$ which describes the availability state of the production system at time $t$, and takes values in $M=\{0,1\} . \alpha(t)=1$, if the manufacturing system is available, and $\alpha(t)=0$ if it is unavailable. 
- A piecewise continuous variable $q(t)$ which describes the lot processing progress (WIP level) at time $t$. Let $0 \leq q(t) \leq Q$ be the capacity constraint of the WIP lot for each $i$ th production cycle.

- A piecewise continuous variable $x(t)$ which describes the final inventory level at time $t$. This quantity faces a continuous downstream demand $d$, a continuous downstream replacement of defective items with good ones while serving the demand, and impulsive upstream supply with variable jump $a^{i} Q$ when the $i$ th lot is received at instant $\xi_{i}$.

- A piecewise continuous variable which describes the inventory position at time $t$ and measured by $y(t), y(t)$ is equal to the instantaneous sum of the stock (inventory/backlog) level $x(t)$ and the total amount of lots-under-sampling and $100 \%$ inspection $\Rightarrow$ $y(t)=x(t)+(S(t)+I(t)) Q$, where $S(t)$ and $I(t)$ are integer variables respectively counting the total number of lots-under-sampling, and the total number of lots-under- $100 \%$ inspection, at each time $t$.

The dynamics of production $q($.$) , inventory position y($.$) and final inventory level x($.$) are given by$ the following difference and differential equations:

$$
\begin{aligned}
& \left.\frac{d q(t)}{d t}=u(t, \alpha), q(0)=q, \forall t \in\right] \theta_{i}, \delta_{i}[\text {, } \\
& q\left(\delta_{i}^{+}\right)=q\left(\delta_{i}^{-}\right)-Q, \\
& \frac{d y(t)}{d t}=\left\{\begin{array}{lll}
-d & \text { if }(x(t) \leq 0) \&(\alpha(t)=0) & y(0)=y, \\
\frac{-d}{1-A O Q(t)} & \text { otherwise } & \forall t \in] \delta_{i}, \delta_{i}+Q \tau_{\text {insp }}[U] \delta_{i}+Q \tau_{\text {insp }}, \delta_{i+1}[,
\end{array}\right. \\
& y\left(\delta_{i}^{+}\right)=y\left(\delta_{i}^{-}\right)+Q \\
& y\left(\left(\delta_{i}+Q \tau_{\text {insp }}\right)^{+}\right)=y\left(\left(\delta_{i}+Q \tau_{\text {insp }}\right)^{-}\right)-\operatorname{Ind}\left(a^{i} \neq 1\right) p^{i} Q, \\
& \frac{d x(t)}{d t}=\left\{\begin{array}{ll}
-d & \text { if }(x(t) \leq 0) \&(\alpha(t)=0) \\
\frac{-d}{1-A O Q(t)} & \text { otherwise }
\end{array}, x(0)=x, \forall t \in\right] \xi_{i}, \xi_{i+1}[, \\
& x\left(\xi_{i}^{+}\right)=x\left(\xi_{i}^{-}\right)+a^{i} Q, \\
& \forall i=1, . ., N
\end{aligned}
$$

where $q, x$ and $y$ respectively denote the WIP level, the inventory position and the finished product inventory level at initial time. $\delta_{i}^{-}$and $\delta_{i}^{+}$denote the left and right boundaries of the $i$ th production run end time $\delta_{i}$ and, $\xi_{i}^{-}$and $\xi_{i}^{+}$denote the left and right boundaries of the arrival time $\xi_{i}$ of the $i$ th lot to the final stock. And $\operatorname{Ind}(\Theta())=.\left\{\begin{array}{l}1 \text { if } \Theta(.) \text { is true } \\ 0 \text { otherwise }\end{array}\right.$. 
Our decision variables are the production rate $u($.$) and a sequence of production orders denoted by$ $\Omega=\left\{\left(\theta_{1}, Q^{1}\right),\left(\theta_{2}, Q^{2}\right), \ldots,\left(\theta_{\infty}, Q^{\infty}\right)\right\}$ where the couple $\left(\theta_{i}, Q^{i}\right)$ represents the production order of the $i$ th lot $Q^{i}$ at time $\theta_{i}$. Let $\Gamma(\alpha)$ denote the set of admissible decisions $(\Omega, u()$.$) given by:$

$$
\Gamma(\alpha)=\left\{(\Omega, u(.)) \mid 0 \leq u(t, \alpha) \leq u_{\max }, 0 \leq q(t, \alpha) \leq Q, 0<Q \leq \min \left\{Q_{\max }^{\text {wip }}, Q_{\max }^{\text {insp }}\right\}\right\}
$$

where $Q_{\max }^{w i p}$ is the maximum WIP storage capacity, and $Q_{\max }^{\text {insp }}$ is the maximum inspection area capacity.

The instantaneous cost function $g($.$) which includes the production, the total WIP stocking, the$ finished product inventory/backlog, and replacement of returned defective items costs is given by the following equation:

$$
\left.g(q(t), x(t), y(t), u(t, \alpha))=C_{\text {hold }}\left(q(t)+y^{+}(t)\right)+C_{\text {back }} x^{-}(t)+C_{\text {prod }} u(t) \quad \forall t \in\right] \delta_{i}, \delta_{i+1}[
$$

where, $y^{+}(t)=\max (0, y(t)), x^{-}(t)=\max (0,-x(t))$.

The instantaneous cost function of the transportation and quality control $R($.$) of lots produced, at$ times $\delta_{i}$ and $\delta_{i}+n \tau_{\text {insp }}(i=1, \ldots, N)$, is given by:

$$
R(Q, \alpha)=\operatorname{Ind}\left(t=\delta_{i}\right) C_{i n s p} n+\operatorname{Ind}\left(t=\delta_{i}+n \tau_{\text {insp }}\right)\left(\begin{array}{l}
C_{t r}+\operatorname{Ind}\left(a^{i}=1\right) C_{r e p} p_{i}(Q-n)+ \\
\operatorname{Ind}\left(a^{i} \neq 1\right)\left(C_{i n s p}(Q-n)+C_{r e j} Q p_{i}\right)
\end{array}\right)
$$

The average cost function of the transportation and quality control $\tilde{R}($.) of one lot can be derived from Eq. (9) as follows :

$$
\tilde{R}(Q, \alpha)=C_{t r}+C_{i n s p} n+P_{a} C_{r e p}(Q-n) \bar{p}+\left(1-P_{a}\right)\left(C_{i n s p}(Q-n)+C_{r e j} Q \bar{p}\right)
$$

Using Eqs. (10) and (11), the expected long-run average cost $J($.$) is written as follows:$

$$
J(q, x, y, \Omega, u, \alpha)=\lim _{T \rightarrow \infty} \frac{1}{T} E\left\{\begin{array}{l|l}
\int_{0}^{T} g(q, x, y, u) d t+\sum_{i=1}^{N(T)} \tilde{R}(Q, \alpha) & \begin{array}{l}
q(0)=q, x(0)=x, \\
y(0)=y, \alpha(0)=\alpha
\end{array}
\end{array}\right\}
$$

The production planning problem considered involves seeking an admissible control policy $\left(\Omega^{*}, u^{*}\right) \in \Gamma(\alpha)$ that minimizes $J($.$) given by (11), considering Eqs. (7), (9) and (10). This is a$ feedback control policy that determines the production orders and the production rate as a function of the system $\operatorname{state}(q, x, y, \alpha)$.

Given the complexity of the system dynamic described by Eqs. (7), and given the high stochastic nature of the problem under study, it is almost impossible to derive an analytical solution for Eq.(11). In such a situation, some computational and numerical approaches (Boukas and Haurie, 1990) have been proposed in the literature to obtain the optimal feedback policy for stochastic and nonlinear control problems, by solving the associated optimality conditions expressed by the HJB equations. These approaches are restricted to small size systems with limited numbers of products, 
machines and states, and cannot be implemented for complex stochastic control problems (Gharbi and Kenné, 2000, 2003).

In the case of the manufacturing system considered in this paper, the HJB equations cannot be obtained due to the randomness of the proportion of defective items, which leads to two major difficulties. First, the delay separating the end of batch production and its arrival at the serviceable stock, is variable because of the variability of the probability of acceptance $P_{a}\left(p_{i}\right), i=1, . ., N$, from lot-to-lot. Second, the real demand rate $d /(1-A O Q(t))$ is also variable because of the variability of the outgoing quality over time. It should be noted that both the delay in quality control and the real demand rate cannot be characterized by a known probability distribution. Therefore, some assumptions used in the literature, such as discretization of the demand distribution into given values (Hu et al., 2004) or giving some specific form to the demand fluctuation (Boukas et al., 2000), cannot be used here. Moreover, the solution of the inventory control problem with uncertain processing delay given by Boukas et al. (2000), which needs difficult calculations to solve some linear matrix inequalities, is not easily implementable to our case. Thus, there is no way to derive the optimality conditions.

Instead of solving Eq. (11) under the constraints of Eqs. (7), either analytically or numerically, we propose, in the next section, an alternative solution based on a heuristic control policy using arguments and approximations.

\section{Heuristic control policy}

In production systems management, one of the main useful strategies for responding to uncertainty is to build a surplus inventory, or safety stock, to hedge against periods in which the production capacity cannot satisfy demand (Hu et al., 2004). For continuous-flow unreliable manufacturing systems, the optimal production policy is of a hedging point policy (HPP) type ((Akella and Kumar, 1986), (Bielecki and Kumar, 1988)). For unreliable batch manufacturing systems, some authors, e.g., Giri and Dohi (2005) and Sana and Chaudhuri (2010), used an optimal safety stock in inventory to protect against possible stock-out during system repair and to enhance customer service levels. In a prior paper, Bouslah et al. (2012) focused on the problem of simultaneously determining the optimal lot sizing and the optimal production control policy of unreliable batch manufacturing systems. Assuming that the system is perfect, they were capable of writing the HJB equations and solving them numerically. They then showed that the optimal feedback control policy is closely approximated by a combination of base-stock policy expressed by a modified HPP and a state dependent economic production quantity policy. When a delay is considered in transporting batches produced to the final stock, the authors assumed that the feedback inventory control is based on the concept of the inventory position, which includes the on-hand inventory in the serviceable stock and the total pending quantities in transportation, as in Mourani et al. (2008) and Li et al. (2009).

Based on the results obtained by Bouslah et al. (2011) in which it was shown that the HPP concept remains optimal for controlling unreliable batch manufacturing systems with delays, and 
considering the effect of quality imperfection on the real demand rate, we propose the following combined control policy :

Production order control policy:

$\Omega^{i}\left(t \geq \delta_{i=1,2, ., \infty}, Q\right)= \begin{cases}Q^{i} & \text { if }\left(\left(y\left(\delta_{i-1}^{+}\right) \leq Z\right) \operatorname{or}\left(y(t)=Z \mid y\left(\delta_{i-1}^{+}\right)>Z\right)\right) \&(\alpha(t)=1) \\ 0 & \text { Otherwise }\end{cases}$

Production rate control policy:

$$
\left.\left.u^{i}(t \in] \theta_{i=1,2, . ., \infty}, \theta_{i+1}\right], \alpha\right)=\left\{\begin{array}{cl}
\alpha(t) u_{\max } & \text { if }\left(\left(y\left(\theta_{i}^{+}\right)<Z\right)\right) \&\left(\theta_{i}<t \leq \delta_{i}\right) \\
\frac{\alpha(t) d}{1-A O Q(t)} & \text { if }\left(y\left(\theta_{i}^{+}\right)=Z\right) \&\left(\theta_{i}<t \leq \delta_{i}\right) \\
0 & \text { if }\left(y\left(\delta_{i}^{+}\right)>Z\right) \&\left(\delta_{i}<t \leq \theta_{i+1}\right)
\end{array}\right.
$$

with the constraints:

$0<Q \leq \min \left\{Q_{\max }^{\text {wip }}, Q_{\max }^{\text {insp }}\right\}, Z \geq 0$.

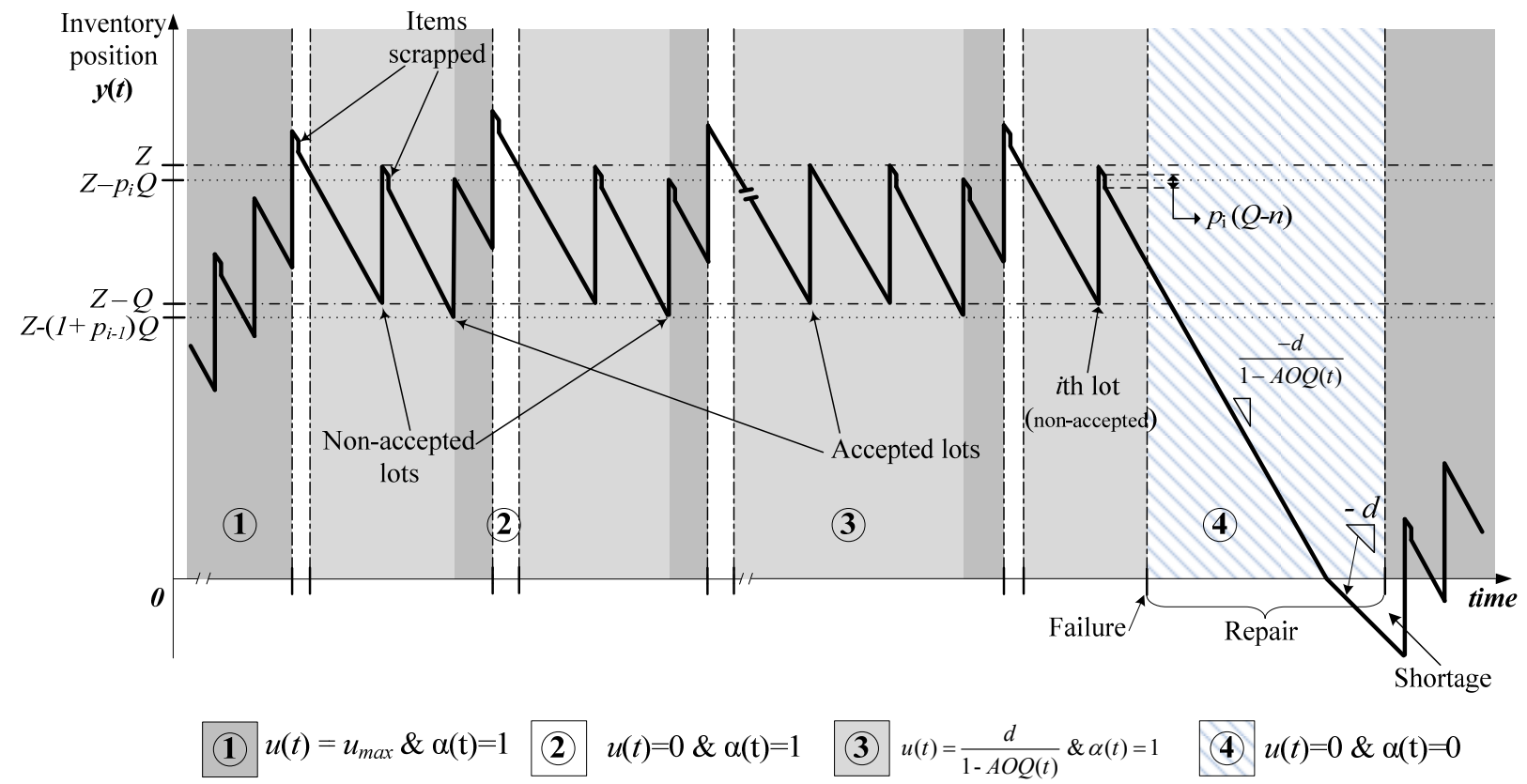

Figure 3. Behaviour of the inventory position over time.

Figure 3 depicts the dynamic of inventory position when the manufacturing system is controlled by the proposed control policy. Indeed, the production order policy is given by the Eq. (12). The decision to produce or not produce a new lot is taken by controlling the inventory position at the end of each production cycle. Three situations can arise:

(i). If at the end of the (i-1)th production cycle $\left(t=\delta_{i-1}^{+}\right)$, the inventory position is less than or equal to the threshold level $Z$, and the system is still available $\left(\alpha\left(\delta_{i-1}^{+}\right)=1\right)$, a new production order $Q^{i}$ is launched (Areas 1 and 3 in Figure 3). In this case, the start time of 
the $i$ th production cycle coincides exactly with the end time of the $(i-1)$ th production cycle $\left(\theta_{i}=\delta_{i-1}^{+}\right)$.

(ii). If at the end of the $(i-1)$ th production cycle, the inventory position is higher than the threshold level $Z$, the production is stopped until the inventory level decreases to the threshold $Z$ (Area 2 in Figure 3). At this time, if the system is still available, a new production order $Q^{i}$ is placed. This time is marked by $\theta_{i}$ and the production of the $i$ th lot starts immediately.

(iii). While the production system is unavailable, no production order is placed (Area 4 in Figure 3)

The production rate control policy is defined by Eq. (13). Note that the production rate of the $i$ th lot can take three possible levels, similarly to the hedging point policy concept. This depends on the inventory position evolution, the instantaneous system availability and the lot processing progress, as follows:

(I). If the inventory position at the beginning of the $i$ th production cycle is strictly below the threshold level $Z$, and while the production system is available $(\alpha(t)=1)$, the corresponding $i$ th lot is manufactured at the maximum production rate $u_{\max }$. Such a case occurs when the production is restarting just after a corrective maintenance or if the (i-2)th lot was rejected. In the last case, note that the result of sampling inspection of the $(i-2)$ th lot (accepted or not) is known at time $\theta_{i-1}+n \tau_{\text {insp }}$, after launching the $(i-1)$ th production order. When the $(i-2)$ th lot is not accepted, the rejection of the $p_{i-2}(Q-n)$ defective items will cause an inventory loss which should be recuperated by accelerating the production in the next $i$ th production order (Area in Figure 3). Note that, in order to ensure the system feasibility condition, the maximum production rate $u_{\max }$ must satisfy the following constraint: $T B F /(T B F+T T R) \cdot u_{\max }>d /(1-A O Q())$.

(II). If the inventory position at the beginning of the $i$ th production cycle is exactly equal to the threshold level $Z$, and while the production system is available $(\alpha(t)=1)$, the production rate of the corresponding $i$ th lot is set instantaneously to the demand rate $(u(t)=d /(1-A O Q(t)))$ in order to maintain the on-hand inventory position (Area 3 in

Figure 3), similarly to the principle of maintaining security stock in classical HPP. If there is no rejection during the $i$ th production cycle, the inventory position at the end of the production cycle is equal to $Z-Q$. If not, the inventory position at the end of the $i$ th production cycle is equal to $Z-\left(1+p_{i-1}\right) Q$. It should be mentioned that from a practical point of view, the instantaneous calculation of the average outgoing quantity $A O Q(t)$ may not be easy because of the operational difficulties of exactly determining the proportion of defective items in each lot produced. In order to facilitate the control policy implementation in practice, we estimate the $A O Q(t)$ by $A O Q(\infty)$, where $A O Q(\infty)=\lim _{t \rightarrow \infty} A O Q(t)$ is the long- 
term average outgoing quality. By replacing the discrete values $p_{i}(\mathrm{i}=1, . ., \infty)$ of the $N(\infty)$ lots produced during the long-term horizon in Eq. (6) by the long-term average proportion of defective items in a lot $\bar{p}$, we obtain :

$$
A O Q(\infty)=\frac{\sum_{i=1 / a^{i}=1}^{N(\infty)} \bar{p}(Q-n)}{\sum_{i=1}^{N(\infty)} a^{i} Q}=\frac{\sum_{i=1 / a^{i}=1}^{N(\infty)} \bar{p}(Q-n)}{\sum_{i=1 / a^{i}=1}^{N(\infty)} Q+\sum_{i=1 / a^{i} \neq 1}^{N(\infty)}(1-\bar{p}) Q}
$$

Given that $\sum_{i=1 / a^{i}=1}^{N(\infty)} 1=P_{a} N(\infty) \sum_{\text {and }}^{N(\infty)} 1=\left(1-P_{a} \neq 1\right) N(\infty)$, we obtain :

$$
A O Q(\infty)=\frac{P_{a}(Q-n) \bar{p}}{Q P_{a}+\left(1-P_{a}\right) Q(1-\bar{p})}
$$

This $\mathrm{AOQ}(\infty)$ formula, Eq. (14), is exactly the theoretical average outgoing quality for given proportion of defective items $\bar{p}$, when the defective items found in the sampling are replaced by good ones and those discovered during 100\% inspection are rejected (see Stephens (2001) and Schilling and Neubauer (2009)).

(III). If the inventory position at the end of the $i$ th production cycle is strictly greater than the threshold level $Z$, the manufacturing is stopped $(u()=0$.$) until the inventory position falls to$ the threshold $Z$ by the effect of the demand and/or rejection of defective items (Area $\mathbf{2}$ in Figure 3). Also, when the production system becomes unavailable $(\alpha(t)=0)$, the production is stopped immediately.

The implementable feedback control process is summarized in Figure 4, which shows the decisions that should be taken at the beginning of each production cycle, depending on the system availability, the quality control results and the position inventory. 


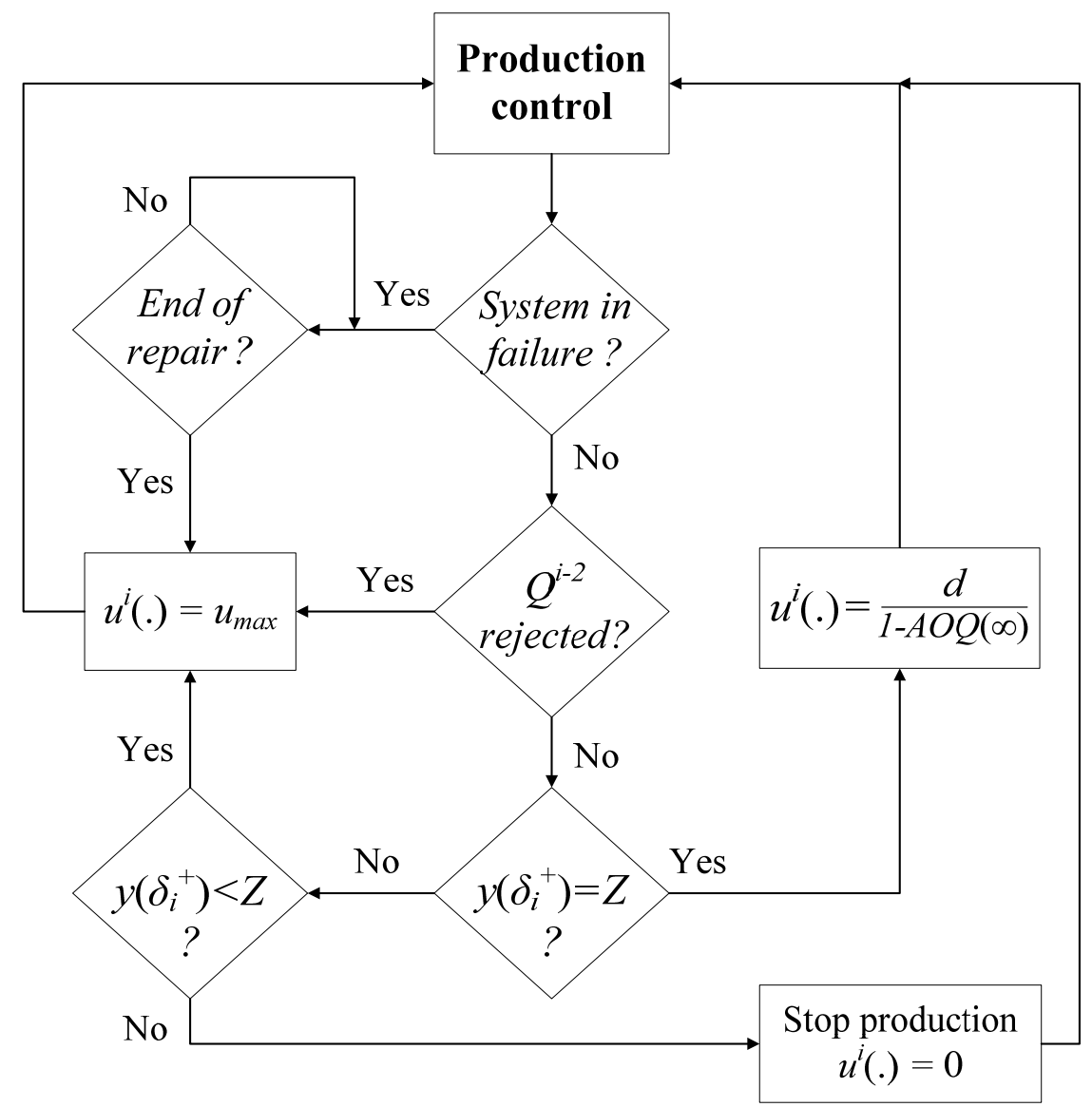

Figure 4. Logigram of the production control process.

\section{Resolution approach}

\subsection{Simulation-based experimental approach}

To optimize the overall expected cost with respect to the design factors $\left(Q^{*}, Z^{*}\right)$, we adopt an experimental approach which combines a simulation model with design of experiments, statistical analysis and response surface methodology. This approach has been used to control diverse problems in manufacturing (Gharbi and Kenné, 2000). It can be applied in our study through the following five steps:

1. Mathematical problem formulation: The objective of this step is to analytically formulate the problem as a stochastic dynamic programming model, as shown in section 2. This provides an understanding of the dynamic of the system as a function of its states, and the calculation of the expected long-run average cost.

2. Heuristic control policy: The heuristic control policy is obtained using theoretical arguments from the literature, as shown in section 3. The proposed policy is used for productioninventory control in the simulation model, and to determine the design factors.

3. Simulation model: The simulation model describes the dynamic of the system using the stochastic dynamic programming model and the heuristic control policy, and evaluates its performances for given factors $(Q, Z)$. These factors are considered as input of such a model, 
and the related incurred cost is defined as its output. For more details concerning the simulation model design, see section 4.2.

4. Design of experiments: The experimental design defines how the control factors $(Q, Z)$ should be varied in order to determine the effects of the mains factors and their interactions (i.e., analysis of variance (ANOVA)) on the incurred total cost through a minimal set of simulation runs.

5. ANOVA, Regression analysis and Response Surface Methodology: A multi-factor statistical analysis (ANOVA) of the simulated data is carried out to show the effects of the design factors $(Q, Z)$, their interaction and their quadratic effects on the response variable (i.e., the cost). Then, the main significant factors and their interactions are considered as input of a regression analysis, which is used in conjunction with the Response surface methodology (RSM), to fit the relationship between the cost and the input factors. RSM is a collection of mathematical and statistical techniques that are useful for modelling and analysing problems in which a response of interest is influenced by several variables and the objective is to optimize this response (Montgomery, 2008).

\subsection{Simulation model}

A combined discrete-continuous model was developed using the SIMAN simulation language with C++ subroutines (Pegden et al., 1995), and then executed through the ARENA simulation software. The advantage of using a combined discrete-continuous model is it reduces the execution time (Lavoie et al., 2007, 2010), and accurately models the impulse-continuous nature of the production-inventory dynamic.

The simulation model can be described following the sequence of numbers appearing in Figure 5, as follows:

(0) Initialization: setting the values of the parameters $\left(u_{\max }, d, n, c, \tau_{\text {insp }}, C_{\text {hold }}, C_{\text {back }}, C_{\text {prod }}, C_{\text {insp }}\right.$, $\left.C_{r e j}, C_{r e p}, \Phi(p), \sim T B F, \sim T T R\right)$, the decision variables $(Q, Z)$, the initial states $(q, x)$ and the simulation run-time $T_{\infty}$. Note that the model is developed to accept any probability distribution for the $\Phi(p), \sim T B F$ and $\sim T T R$.

(1) Network (II) models failure and repair events. The system starts at an initial time with an operational state. The entity here circulates in a loop model and updates the system availability state, depending on its position in the model: $\alpha(t)=1$ if the entity holds in $T B F$ delay block, and $\alpha(t)=0$ if it holds in TTR delay block.

2 In network (I), the production order and production rate control are performed according to the control policy (Eqs. (12) and (13)). The entity here represents the lot to be produced.

3 The production processing delay is determined by dividing the lot sizing $Q$ by the corresponding production rate $u^{i}$ (.).If the system becomes unavailable, the production is interrupted using the pre-emption mechanism of SIMAN until the end of the system repair. When the lot processing is completed, the original entity is sent back to the production control blocks (point 2 ) and a duplicated entity is created and sent to an assignment block where the WIP lot level and the inventory position are updated using difference equations in Eqs. (7). 
(4) A discrete proportion of defective items is attributed randomly to each lot produced following the probability function $\Phi(p)$ and the associated probability of acceptance $P_{a}($.$) is calculated using$ Eq.(4) as in Stephens (2001) and Besterfield (2009). Then, the entity (lot produced) holds in a DELAY block for inspection.

(5) The decision to accept or reject the lot is modeled by a probabilistic BRANCH block of SIMAN, in order to ensure that $P_{a}$ lots inspected are accepted, and $\left(1-P_{a}\right)$ lots are rejected. Rejected lots hold in an additional DELAY block for $100 \%$ inspection.

6 When a lot is accepted, the attribute $a^{i}$ is set to 1 and the total cumulative quantity of returned/replaced defective items, expressed by the discrete variable Return, is updated. When a lot is rejected, the attribute $a^{i}$ is set to $1-p_{i}$, the cumulative rejected quantity of defective items, expressed by the discrete variable Reject, is updated, and the rejected defective items are subtracted from the inventory position.

7 When a lot arrives in the serviceable final stock, the corresponding entity impulsively updates the inventory level as in Eqs. (7). A discrete variable $A I($.$) is defined to calculate the cumulative$ quantity entering the final stock during the simulation run. The average outgoing quantity $A O Q($. is then updated using Eq. (11), with $A I($.) used as a denominator.

8 Simulation run-time control: if the current time $T N O W$ exceeds the predefined simulation runtime $T_{\infty}$, the simulation run is stopped. Otherwise, the duplicated entity disappears at this stage.

$9 \mathrm{C}++$ routines are used to model the dynamic of production and inventories as described in the differential equations in Eqs. (7).

$10 / 11 / 12$ Then, the WIP level $q($.$) , the inventory position y($.$) , and the inventory level x($.$) are$ integrated continuously, using the Runge-Kutta-Fehlberg (RKF) method, which guarantees consistent accuracy, as mentioned in Pegden et al. (1995).

13 This routine allows an instantaneous calculation of the surplus and backlog levels.

14 At the end of the simulation run, the total expected cost $J($.$) is calculated using Eq. (11).$ 


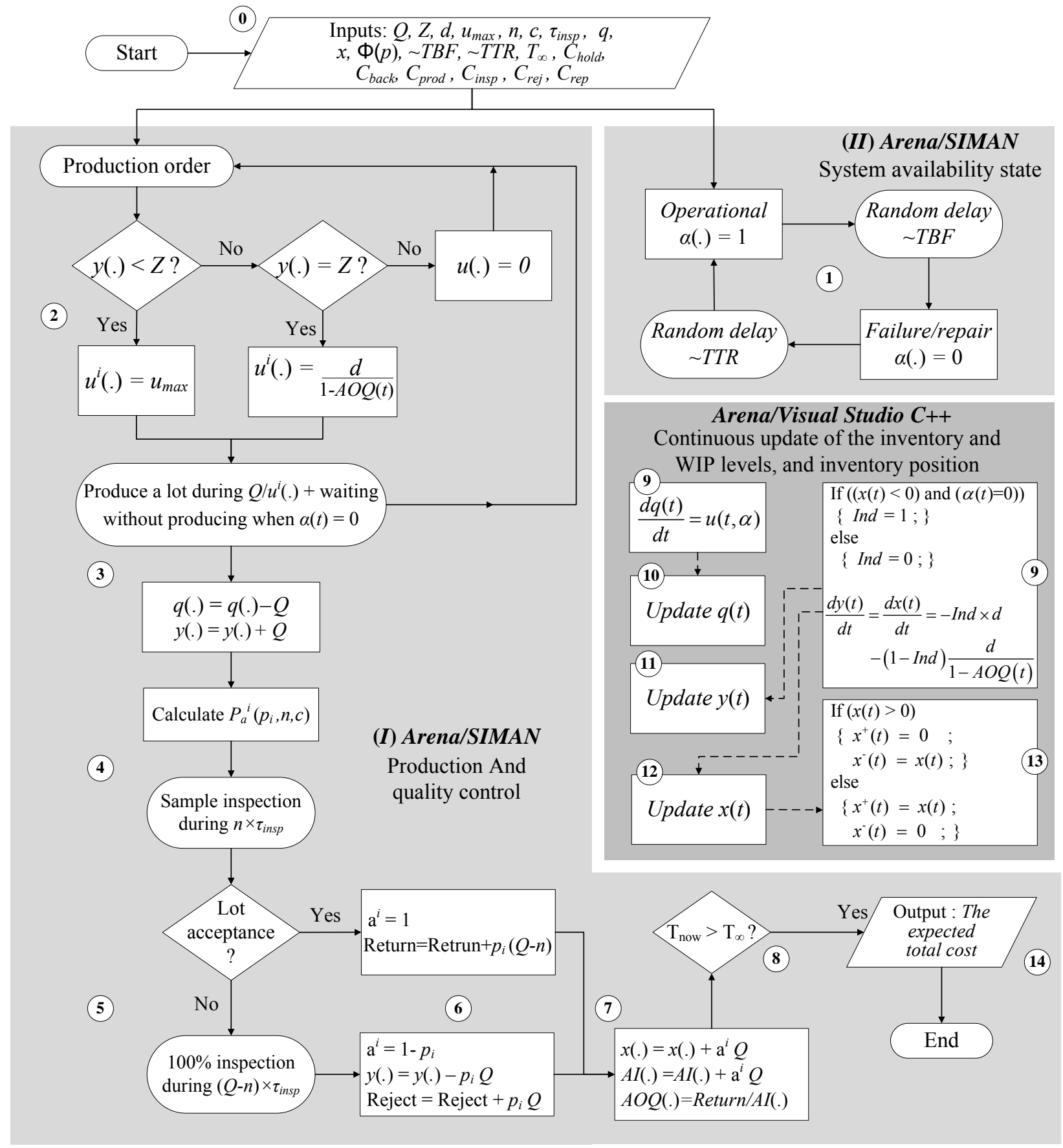

Trajectories of entities in the discrete networks.

Figure 5. Simulation diagram.

\subsection{Validation of the simulation model}

To verify the accuracy of the simulation model, we graphically examine the trajectories of the production rate and the inventory position during the simulation run. Figure 6 shows that the model performs correctly as expected and intended, and adequately represents the control policy: 
A new production cycle is launched only when the inventory position is less than or equal to the threshold $Z$, as described in Eq. (12). The production rate value changes instantaneously in response to changes in the inventory position and the system availability state as described in Eq. (13). We also verify that when a lot is rejected, a next lot is manufactured with the maximum production rate in order to recuperate the loss of inventory (scrapped items) and therefore maintain the inventory position around the threshold $Z$. The impact of the $100 \%$ inspection delay is clearly shown on the time lag of the inventory level trajectory $x(t)$ as compared with the inventory position trajectory $y(t)$ as described in section 2.2 .

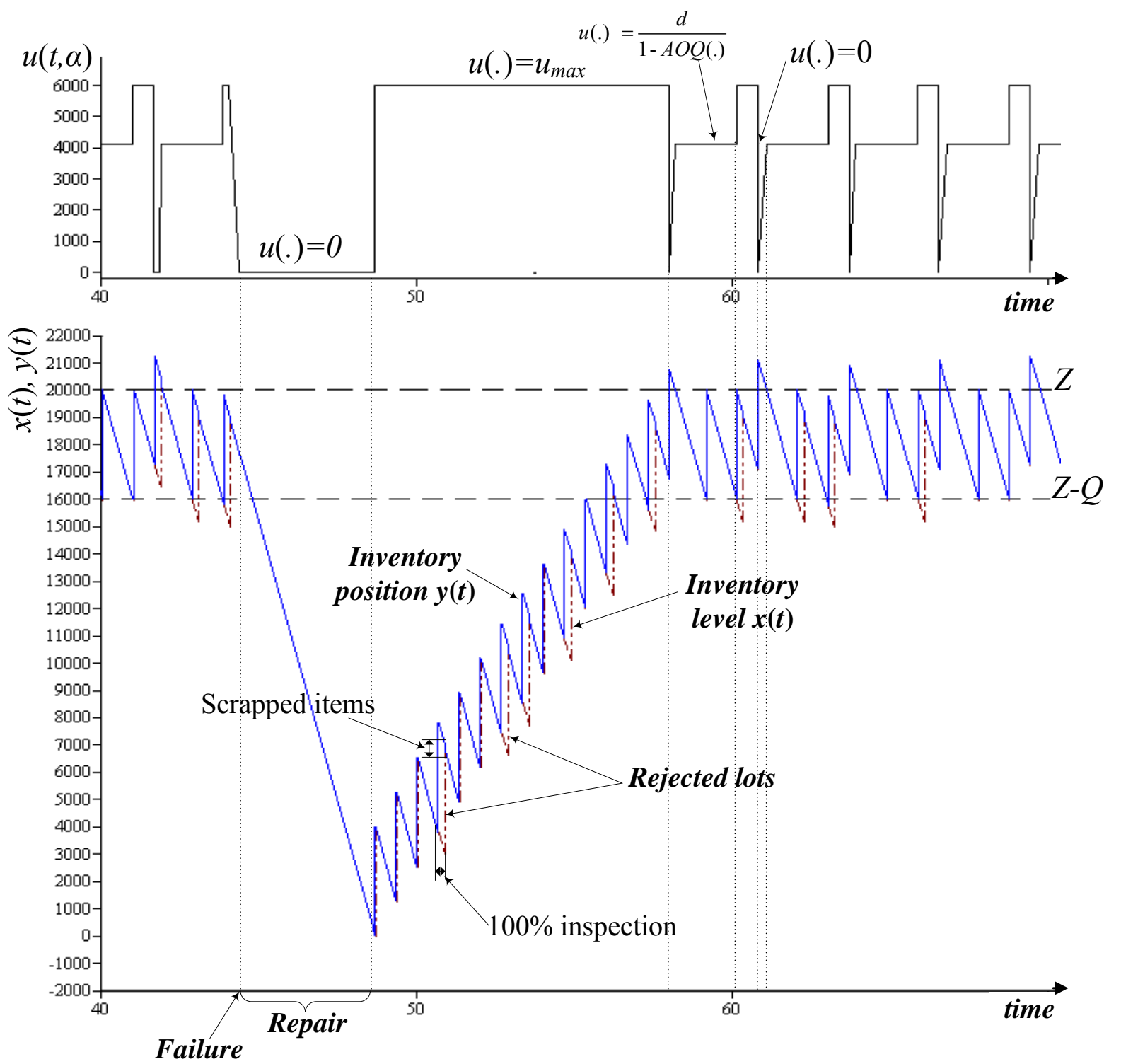

Figure 6. Production rate and inventory position evolution during simulation run.

\section{Numerical example}

In this section, we present a numerical example to illustrate the simulation-based experimental approach. The following parameters are considered in appropriate units: $u_{\max }=6000, d=4000$, 
$\Phi(p)=\operatorname{Uniform}(0.03,0.06), \sim T B F=\log -\operatorname{Normal}(50,5), \sim \operatorname{TTF}=\operatorname{Gamma}(0.5,10), \tau_{\text {insp }}=5 \times 10^{-5}$

, $n=48, c=2, C_{\text {hold }}=0.1, C_{\text {back }}=1.5, C_{\text {tr }}=1500, C_{\text {prod }}=0.25, C_{\text {insp }}=0.5, C_{r e j}=5, C_{r e p}=7.5$.

Simulation runs are conducted according to a complete $3^{2}$ experiments plan with five replications for each combination of factors $(Q, Z)$. This requires $45\left(3^{2} \times 5\right)$ simulation runs. In order to ensure that the steady-state is reached, the duration of each simulation run is set such as to observe 10,000 failures in each replication, i.e., $T_{\infty}=500,000$ units of time. The selection of the levels of the experimental design plan parameters is an important factor in the precision of the response surface. It should be precise enough so that the response surface estimates the total expected cost function accurately, but large enough so that the effect of the parameters is not hidden by the inherent variability of the response. In order to select these levels correctly, we repeat the design of experiments (DOE), simulation and optimization using RSM sequence, narrowing the domain of $(Q, Z)$ around the last found solution until it is centered about the optimum design point. Through this sequential procedure, the admissible experimentation region is explored and therefore the obtained solution will be a global optimum.

In order to reduce the variability in the results from one configuration of the DOE to another, we use the common random number technique (Law \& Kelton, 2000) which consists in submitting different configurations to identical scenarios to estimate the response of the system under similar quality imperfection state and failure and repair events. This allows us to remove a portion of the variability in the response that is attributed to the particular series of random events.

The data simulation is carried out using statistical software (STATISTICA) to seek a regression model fitting the response variable (total expected cost). We assume here that a continuous function $\tilde{J}($.) of $Q$ and $Z$ exists, fitting a second-order regression model and relating the response variable to the design factors. The function $\tilde{J}($.$) is called the response surface and takes the$ following equation:

$$
\tilde{J}(Q, Z)=\beta_{0}+\beta_{1} Q+\beta_{2} Z+\beta_{12} Q Z+\beta_{11} Q^{2}+\beta_{22} Z^{2}+\varepsilon
$$

where, $\beta_{0}, \beta_{i}(\mathrm{i}=1,2), \beta_{12}, \beta_{i i}(\mathrm{i}=1,2)$ are unknown parameters to be estimated from the collected simulation data, and $\varepsilon$ is a random error. It should be noted that the idea of approximating the function cost by quadratic model has been widely used in the literature (see, for example, Noguera \& Watson (2006), Lavoie et al. (2010)). The significant effects are provided through a multi-factor analysis of variance (ANOVA), and the regression model is then determined using the response surface methodology.

To check the suitability of the second-order regression model in the local region of the optimal solution, we use three ways as in Myers et al. (2009), Noguera \& Watson (2006) and Lavoie et al. (2010), as follows:

1) The model's overall performance is evaluated. This is referred to as the coefficient of multiple determination R-squared and the adjusted R-squared which represent the proportion of total 
variation explained by the second-order regression model. The values of these two coefficients should be close to 1.0.

2) A complete residual analysis is done to check the normality assumption and the homogeneity of residuals.

3) Once the optimization is performed, the solution is cross-checked to ensure the validity. In fact, $Q^{*}$ and $Z^{*}$ are used as input to the simulation model and we verify that the obtained optimal cost $\tilde{J}\left(Q^{*}, Z^{*}\right)$ should fall in the Confidence Interval $\left(\bar{J}(m) \pm t_{m-1,1-\alpha / 2} S(m) / \sqrt{m}\right)$, obtaining using $m$ replications of simulation. Where: $\bar{J}(m)$ is the average cost obtained from the $m$ replications; $t_{m-1,1-\alpha / 2}$ is the student coefficient function of $m$ and $\alpha$ with $(1-\alpha)$ the confidence level (set at 95\%); and $S(m)$ is the sample standard deviation.

By applying the sequential procedure of RSM, the levels of the independent variables have been chosen as in Table 1.

Table 1. ANOVA table for the total cost.

\begin{tabular}{lccll}
\hline Factor & Low level & Center & High level & \multicolumn{1}{c}{ Description } \\
\hline$Q$ & 7000 & 9500 & 12000 & Lot size \\
$Z$ & 21000 & 25500 & 30000 & Hedging level \\
\hline
\end{tabular}

Table 2 summarizes the ANOVA of the collected data. For each design factor (including the linear and the quadratic effects) and its interaction, the table presents the Sum of Squares (SS), the degree of freedom (d.f.), the Mean Square (MS), an F-ratio, computed using the residual mean square, and the significance level of the P-value. We can see that the linear and quadratic effects of the two factors $(Q, Z)$ and their interaction $Q . Z$ are significant for the dependent variable (symbol Yes in the last column), at the 0.05 level of significance.

Table 2. ANOVA table for the total cost.

\begin{tabular}{lrrrrrc}
\hline Factor & SS & d.f. & MS & F-Ratio & P-value & Significant \\
\hline$Q$ (Linear + quadratic) & 49418.0 & 2 & 24709.0 & 64.0734 & 0.000000 & Yes \\
$Z$ (Linear + quadratic) & 149854.7 & 2 & 74927.3 & 194.2956 & 0.000000 & Yes \\
$Q . Z$ & 168796.1 & 1 & 168796.1 & 437.7088 & 0.000000 & Yes \\
Error & 15039.8 & 39 & 385.6 & & & \\
Total SS & 383108.6 & 44 & & & & \\
\hline $\mathrm{R}^{2}=0.9632, \mathrm{R}^{2}$-Adjusted= $=0.9585$ & & & & &
\end{tabular}

The R-squared value of 0.9632 and the adjusted R-squared value of 0.9585 presented in Table 1 states that about of $96 \%$ of the observed variability in the total expected cost is explained by the model (Montgomery, 2008). A residual versus fitted values plot and a normal probability plot of residuals were used to check the homogeneity of the variances and the residual normality, respectively. This therefore confirmed that the total expected cost $\tilde{J}($.) can be fitted by a secondorder polynomial model. From STATISTICA, the corresponding quadratic function is given by: 


$$
\tilde{J}(Q, Z)=9417.20-5.59 \times 10^{-3} Q-229.96 \times 10^{-3} Z-8.17 \times 10^{-6} Q Z+11.25 \times 10^{-6} Q^{2}+6.04 \times 10^{-6} Z^{2}
$$

Figure 7 presents the projection of the cost response surface (16) on a two-dimensional plan $(Q$, $Z$ ). The minimum total expected cost $\tilde{J}^{*}()=$.6465.32 is located at $Q^{*}=9485$ and $Z^{*}=25443$ as shown in Figure 7. These values represent the best parameters of the heuristic control policy which should be applied to control both the production order and the production rate. From 30 replications of simulation, we validated the solution by verifying that the estimated optimal cost $\tilde{J}^{*}()=$.6465.32 is within the $95 \%$ Confidence Interval [6464.40, 6473.80].

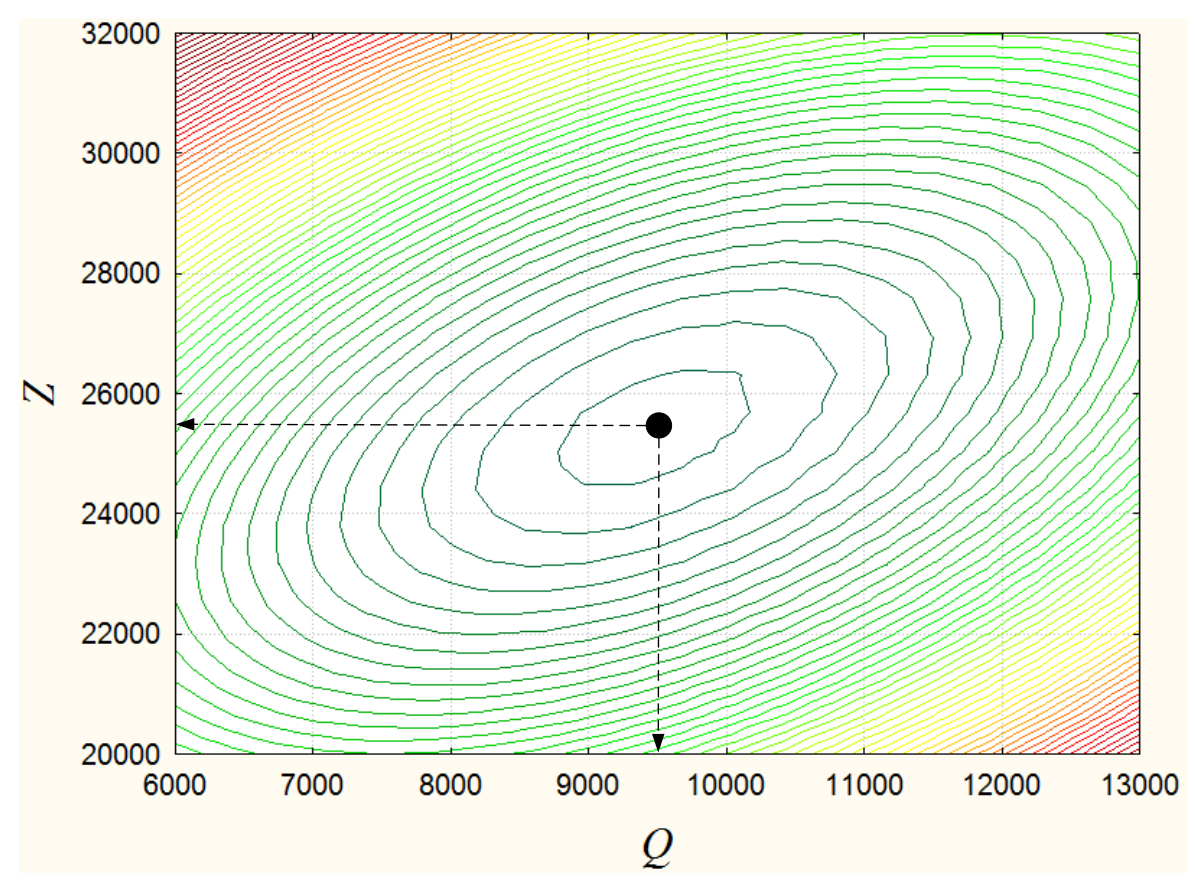

Figure 7. Contour plot of the cost function surface.

\section{Sensitivity analysis}

\subsection{Sensitivity analysis of cost parameters}

A sensitivity analysis of the control policy is conducted with respect to cost parameters in order to prove the efficiency of the heuristic control policy and the robustness of the resolution approach. Table 3 presents ten configurations of cost parameters derived from the basic case, and their impact on the optimal design factors and incurred cost. The results obtained make sense, as expected, and can be explained as follows:

- Variation of the inventory cost: When the inventory cost increases (case 2), the optimal hedging threshold $Z^{*}$ decreases in order to avoid further inventory costs. In addition, the optimal lot sizing $Q^{*}$ decreases to ensure a better supply to the final stock against the risk of shortages becoming higher. The decrease in inventory cost (case 1) produces the opposite effects.

Table 3. Sensitivity analysis for cost parameters. 


\begin{tabular}{cccccccccc}
\hline Case & $C_{\text {hold }}$ & $C_{\text {back }}$ & $C_{\text {insp }}$ & $C_{\text {rej }}$ & $C_{\text {rep }}$ & $Q^{*}$ & $Z^{*}$ & Optimal cost & Impact \\
\hline Basic & $\mathbf{0 . 1}$ & $\mathbf{1 . 5}$ & $\mathbf{0 . 5}$ & $\mathbf{5}$ & $\mathbf{7 . 5}$ & 9485 & 25443 & 6465.32 & - \\
1 & $\mathbf{0 . 0 5}$ & 1.5 & 0.5 & 5 & 7.5 & 11762 & 30735 & 5227.08 & $\mathrm{Q} \uparrow \quad \mathrm{Z} \uparrow$ \\
2 & $\mathbf{0 . 1 5}$ & 1.5 & 0.5 & 5 & 7.5 & 7423 & 20534 & 7466.77 & $\mathrm{Q} \downarrow \mathrm{Z} \downarrow$ \\
3 & 0.1 & $\mathbf{0 . 7 5}$ & 0.5 & 5 & 7.5 & 10065 & 18691 & 6021.95 & $\mathrm{Q} \uparrow \mathrm{Z} \downarrow$ \\
4 & 0.1 & $\mathbf{2 . 2 5}$ & 0.5 & 5 & 7.5 & 9119 & 27767 & 6683.10 & $\mathrm{Q} \downarrow \mathrm{Z} \uparrow$ \\
5 & 0.1 & 1.5 & $\mathbf{0 . 2 5}$ & 5 & 7.5 & 9565 & 25526 & 6081.82 & $\mathrm{Q} \leftrightarrow \mathrm{Z} \leftrightarrow$ \\
6 & 0.1 & 1.5 & $\mathbf{0 . 7 5}$ & 5 & 7.5 & 9608 & 25552 & 6853.75 & $\mathrm{Q} \leftrightarrow \mathrm{Z} \leftrightarrow$ \\
7 & 0.1 & 1.5 & 0.5 & $\mathbf{2 . 5}$ & 7.5 & 9592 & 25543 & 6285.66 & $\mathrm{Q} \leftrightarrow \mathrm{Z} \leftrightarrow$ \\
8 & 0.1 & 1.5 & 0.5 & $\mathbf{7 . 5}$ & 7.5 & 9582 & 25535 & 6649.91 & $\mathrm{Q} \leftrightarrow \mathrm{Z} \leftrightarrow$ \\
9 & 0.1 & 1.5 & 0.5 & 5 & $\mathbf{5}$ & 9596 & 25552 & 6180.65 & $\mathrm{Q} \leftrightarrow \mathrm{Z} \leftrightarrow$ \\
10 & 0.1 & 1.5 & 0.5 & 5 & $\mathbf{1 0}$ & 9577 & 25527 & 6754.92 & $\mathrm{Q} \leftrightarrow \mathrm{Z} \leftrightarrow$ \\
\hline
\end{tabular}

- Variation of backlog cost: When the backlog cost increases (case 4), more safety stock should be held in order to provide better protection to the system against shortages, which explains the increase in the optimal hedging threshold $Z^{*}$. The optimal lot size decreases in order to reduce the production delay and $100 \%$ inspection delay for rejected lots, and therefore ensure better supply to the final stock. The decrease in backlog cost (case 3) produces the opposite effects.

- Variation of quality costs: We notice that quality costs (inspection, rejection and replacement costs) do not have significant effects on the optimal control policy parameters $\left(Q^{*}, Z^{*}\right)$. From a theoretical point of view, this can be explained as follows: the total expected cost of inspection $T E C_{i n s p}$, rejection $T E C_{\text {rej }}$ (for non-accepted lots), and replacement of returned defective items $T E C_{\text {rep }}$ (for accepted lots), can be estimated using the following formulas, respectively:

$$
\begin{aligned}
T E C_{\text {insp }} & =\frac{C_{\text {insp }} \cdot A T I \cdot N\left(T_{\infty}\right)}{T_{\infty}} \\
T E C_{r e j} & =\frac{C_{r e j}\left(1-P_{a}\right) \bar{p} Q N\left(T_{\infty}\right)}{T_{\infty}} \\
T E C_{\text {rep }} & =\frac{C_{r e p} P_{a} \bar{p}(Q-n) N\left(T_{\infty}\right)}{T_{\infty}}
\end{aligned}
$$

where, $A T I$ is the average total inspection per lot $\Rightarrow A T I=n+\left(1-P_{a}\right)(Q-n)$ (see Besterfield, 2009).

Given that the quantity $Q N\left(T_{\infty}\right)$ in the three formulas (17), (18) and (19) is the constant total amount of items to be produced during $T_{\infty}$ to face the continuous demand, it can be understood that the change in each unit quality cost does not have a significant impact on the optimal lot size $Q^{*}$. Moreover, from these formulas, it is clear that the total quality costs $\left(T E C_{i n s p}, T E C_{r e j}\right.$ and 
$\left.T E C_{r e p}\right)$ do not depend on the inventory state, which explains the fact that there is no change in the threshold $Z^{*}$ when varying the unit quality costs.

Through the above analysis, we conclude that the results make sense. In the next section, we study the effects of sampling plan, quality deterioration and inspection delay on the optimal parameters $\left(Q^{*}, Z^{*}\right)$.

\subsection{Sensitivity analysis of sampling plan severity}

Another set of experiments was conducted with respect to inspection severity in order to study the effect of the choice of such sampling plan on the optimal parameters $\left(Q^{*}, Z^{*}\right)$. Note that, for a fixed acceptance number $c$, when the sample size $n$ increases, the inspection becomes more severe, and therefore, the probability of acceptance of a lot $P_{a}$ decreases (for more details see Besterfield (2009)). It should be noted that if the $P_{a}$ decreases (increases), the average outgoing quality $A O Q$ and the real demand rate decrease (increase) accordingly. Table 4 presents the average probability of acceptance, the long-term $A O Q$, and the demand rate for three degrees of inspection severity: a normal inspection plan that has been used in the previous numerical example with parameters $n=48$ and $c=2$, a reduced sampling plan with $n=32$ and $c=2$, and a tightened sampling plan with $n=86$ and $c=2$.

Table 4. Characteristics of the three degrees of inspection severity.

\begin{tabular}{lccc}
\hline Inspection severity & $P_{a}(\bar{p})$ & $A O Q(\infty)$ & $d /(1-\operatorname{AOQ}(\infty))$ \\
\hline Reduced $(n=32, c=2)$ & 0.824 & $3.68 \%$ & 4152.88 \\
Normal $(n=48, c=2)$ & 0.633 & $2.83 \%$ & 4116.54 \\
Tightened $(n=86, c=2)$ & 0.258 & $1.15 \%$ & 4046.62 \\
\hline
\end{tabular}

When the inspection is tightened (reduced), the optimal overall cost $J^{*}($.$) increases/decreases,$ depending on the balance between $100 \%$ inspection and rejection costs and replacement cost; the optimal lot size $Q^{*}$ decreases (increases); and the optimal hedging threshold $Z^{*}$ increases/decreases, depending essentially on the balance between replacement and shortage costs. 


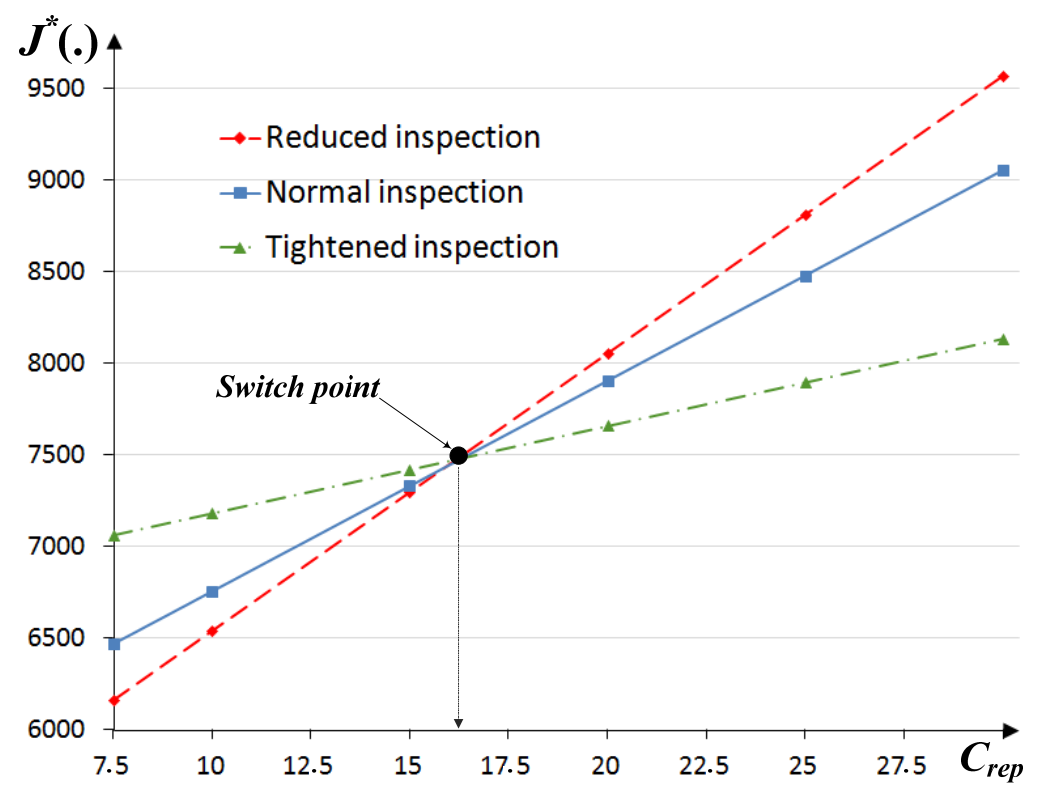

Figure 8. Optimal total expected cost comparison with different replacement costs.

Let us start with Figure 8, which illustrates the variations in the incurred costs for the three types of inspection (reduced, normal and tightened) when varying the unit replacement $\operatorname{cost} C_{\text {rep }}$. Given that more lots are rejected under tightened inspection, the $100 \%$ inspection cost and defective items rejection cost increase, and consequently, the overall cost increases as well. However, for a higher unit replacement cost, the overall cost of reduced inspection becomes greater than that under tightened inspection because the outgoing quality is higher, which causes a higher replacement cost for defective items returned from consumer. We have a single unit replacement cost point, at which the optimal overall expected cost $J^{*}($.$) is the same whatever the inspection$ severity. This corresponds to a switching point separating two types of the optimal overall cost variation, as explained earlier.

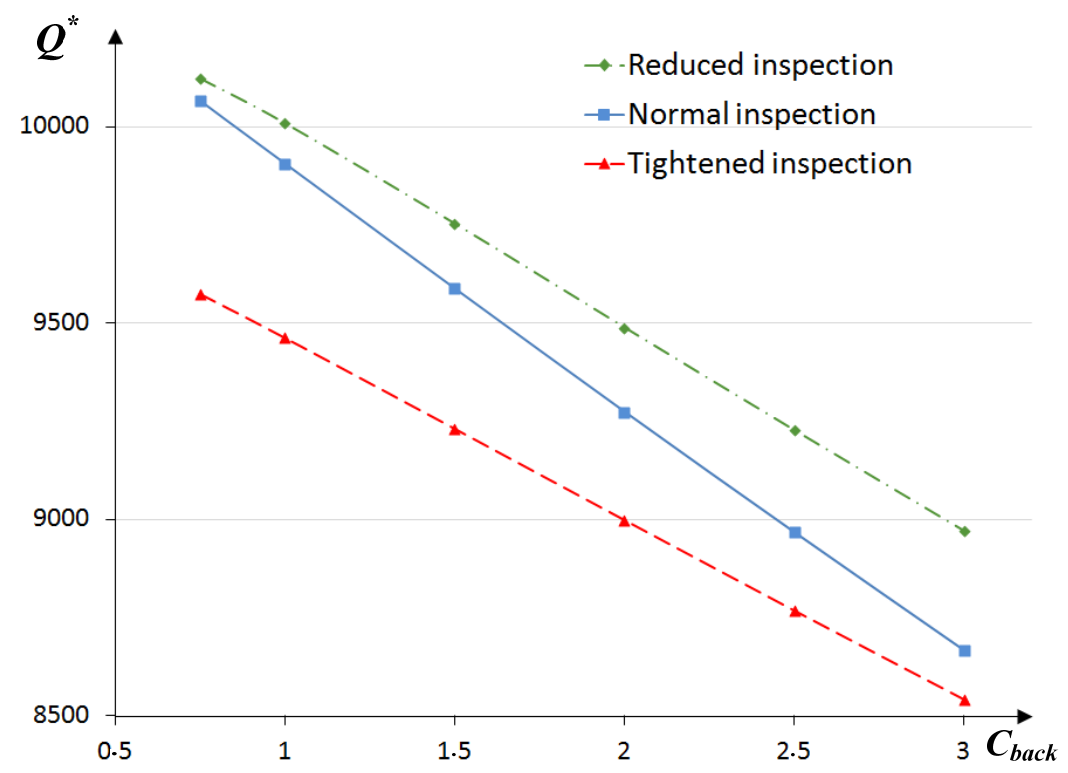

Figure 9.a. Optimal lot size comparison with different backlog costs. 


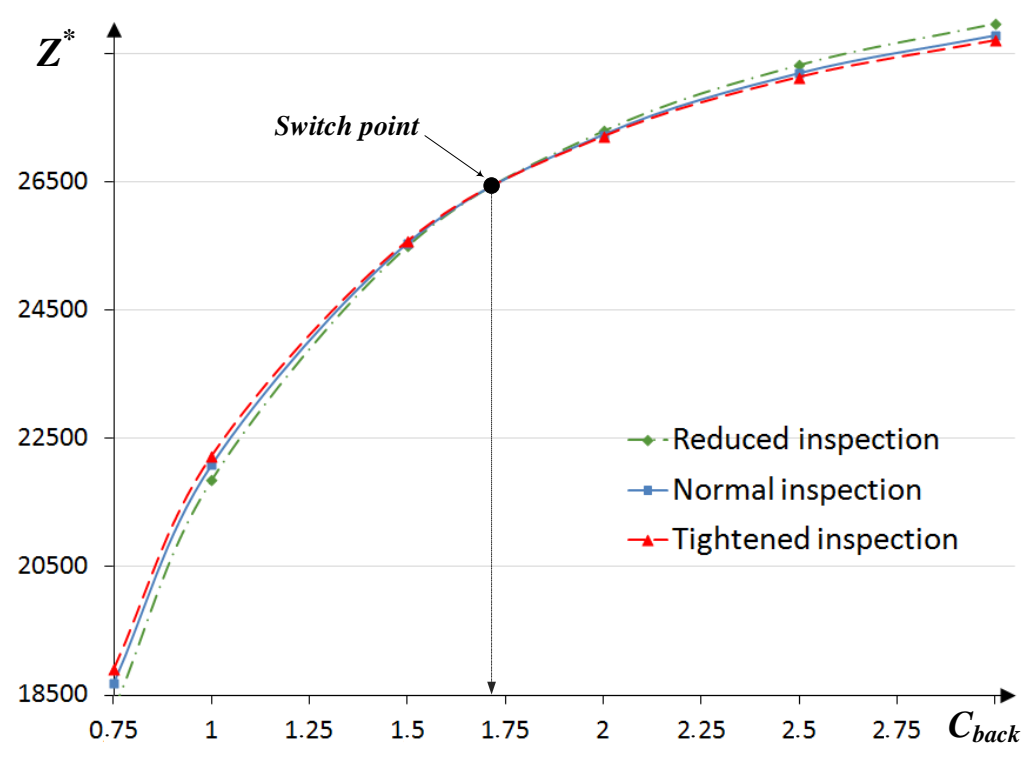

Figure 9.b. Optimal hedging threshold comparison with different backlog costs.

Figures 9.a and 9.b show the effects of inspection severity on the optimal parameters $\left(Q^{*}, Z^{*}\right)$ when varying the unit backlog cost:

- When we tighten the inspection, more lots are rejected and receive $100 \%$ inspection, before being entered into the final inventory. In this case, the system reacts by reducing the optimal lot size $Q^{*}$ in order to reduce production and $100 \%$ inspection delays, and therefore ensure better supply to the serviceable stock. Reduced inspection produces the opposite effect.

- When we tighten the inspection, the optimal threshold $Z^{*}$ increases to reduce shortage risk becoming higher as explained in the previous point. However, for higher units of backlog costs, we notice that there is a common switching point for the three degrees of inspection severity. In contrast with the case with a lower backlog cost, the optimal threshold $Z^{*}$ under reduced inspection becomes greater than that under tightened inspection, in order to meet the higher demand rate and to minimize the shortage cost. It should be recalled here that the downstream demand rate under reduced inspection is higher than that under normal or tightened inspection, as shown in Table 3.

\subsection{Sensitivity analysis of the proportion of defective items}

Additional experiments were conducted to study the impact of quality imperfection of the production system on the optimal control policy parameters $\left(Q^{*}, Z^{*}\right)$. First, we note that the $A O Q$ curve with respect to $\bar{p}$ starts at 0 when $\bar{p}=0$ (no lots rejected), rises to a maximum percentage called the Average Outgoing Quality Limit $A O Q L$ with respect to a particular proportion of defective items $p_{A O Q L}$, and then declines as more and more of the lots produced are rejected (Schilling and Neubauer, 2009). 


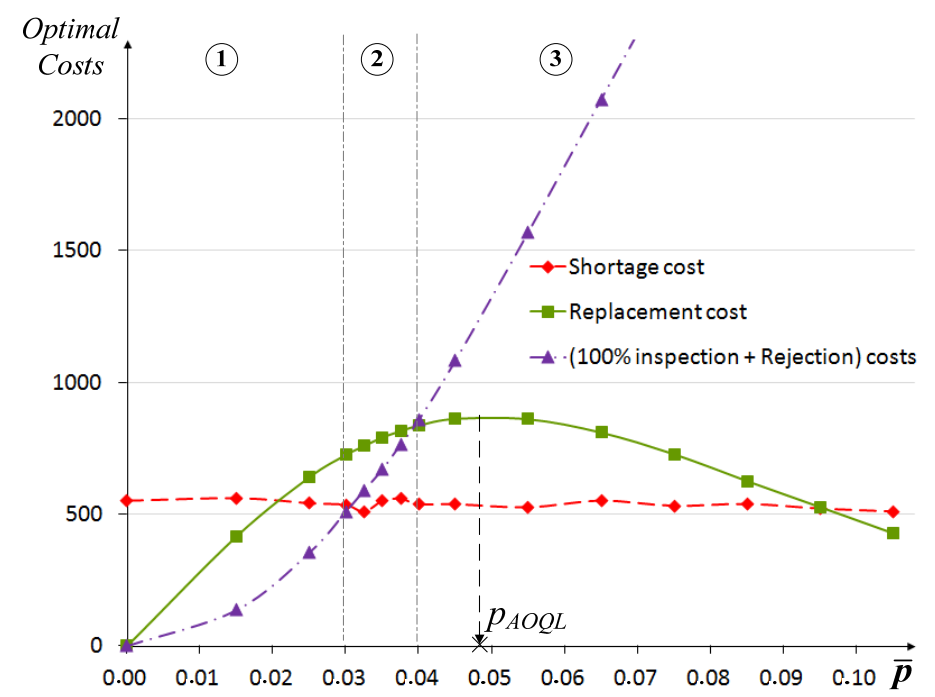

Figure 10.a. Effect of the percentage of defective items on the optimal partial costs.

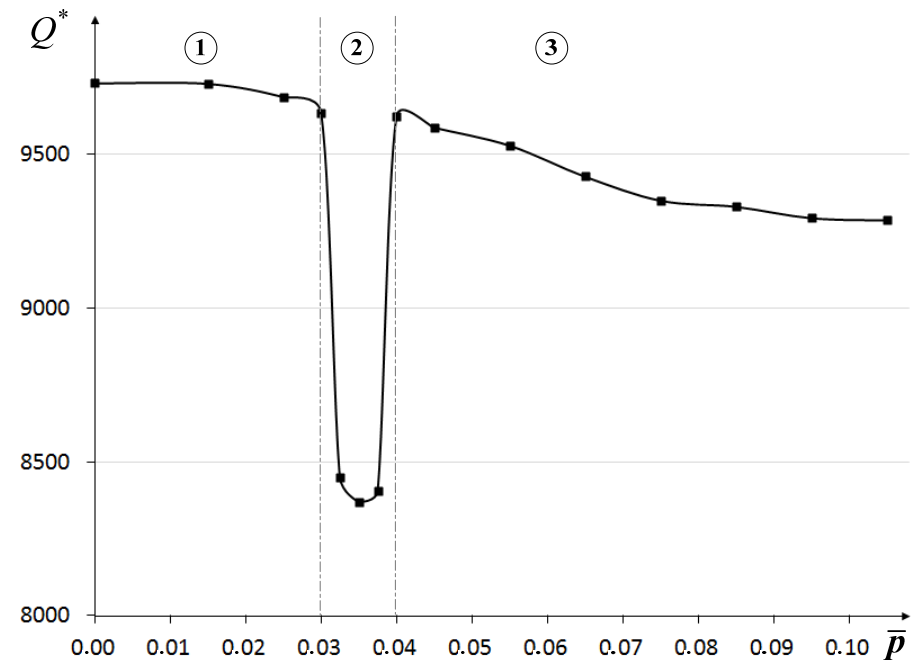

Figure 10.b. Effect of the percentage of defective items on the optimal lot size $Q^{*}$.

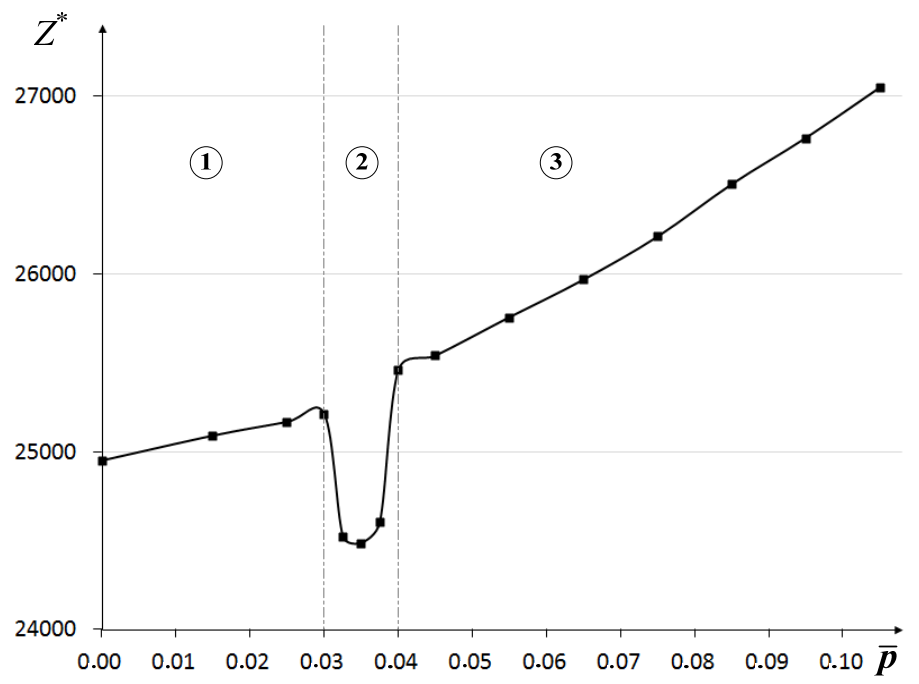

Figure 10.c. Effect of the percentage of defective items on the optimal threshold $Z^{*}$. 
Figure 10.a shows the behaviour of some optimal partial costs with respect to $\bar{p}$ : the replacement cost for returned defective items has a similar behaviour as the $A O Q$ curve, with the $100 \%$ inspection and rejection costs increasing considerably with an increase in $\bar{p}$, and the shortage cost being remaining approximately constant. Figures $10 . \mathrm{b}$ and 10.c show three principal regions of the optimal parameters $\left(Q^{*}, Z^{*}\right)$ variations with respect to $\bar{p}$ :

- In region 1 : When the proportion of defective items increases, the probability of acceptance $P_{a}(\bar{p})$ decreases (Besterfield, 2009), and the number of rejected lots which need $100 \%$ inspection, increases accordingly. Consequently, the optimal hedging threshold $Z^{*}$ increases and the optimal lot size $Q^{*}$ decreases in order to reduce the shortage risk becoming higher.

- In region (2): A particular phenomenon appears on the balance between partial costs. If we take another look at Figure 10.a, we see that when the $A O Q$ approaches the maximum level of $A O Q L$, particularly in region 2 , the replacement cost is greater than the backlog cost, and relatively greater than the sum of the $100 \%$ inspection and rejection costs. In this situation, the optimal threshold $Z^{*}$ falls conversely to its variation sense in region (1), and then resumes its increase. Indeed, the system reacts by reducing the on-hand serviceable inventory containing a high percentage of defective items in order to avoid further replacement cost of the returned items. In other words, it is preferable to have a higher shortage risk than to buy a high level of defective items to consumer with high replacement cost. The optimal lot size $Q^{*}$ decreases and then increases, following the variation of the optimal threshold $Z^{*}$, in order to provide more protection to the final stock against shortage.

- In region 3 : When the sum of $100 \%$ inspection and rejection costs becomes greater than the replacement cost because of an increase in the number of lots rejected, the optimal lot size $Q^{*}$ and the optimal threshold $Z^{*}$ resume their variations, as in region 1 , for the same reasons explained in the first point.

\subsection{Sensitivity analysis of inspection delay}

In this section, we study the sensitivity of the optimal control policy parameters $\left(Q^{*}, Z^{*}\right)$ to changes in inspection delays. Table 5 presents the optimal parameters $\left(Q^{*}, Z^{*}\right)$ and the incurred cost variations corresponding to different inspection delay variations.

When the inspection delay increases (cases (c) and (d)), the optimal lot size $Q^{*}$ decreases and the optimal hedging threshold $Z^{*}$ increases. Indeed, when the lots rejected require a lot of time for $100 \%$ inspection, the shortage risk becomes higher, which explains the increase in the optimal threshold. The optimal lot size decreases in order to reduce inspection delays per lot, and to ensure better supply to the final stock. The optimal overall cost increases due to the increased transportation costs. Obviously, the decrease in inspection delays (cases (a) and (b)) produces the opposite effects. 
Table 5. Sensitivity analysis for the inspection delay.

\begin{tabular}{lccccc}
\hline Case & $\tau_{\text {insp }}\left(\times 10^{-5}\right)$ & $Q^{*}$ & $Z^{*}$ & Optimal Cost & Impact \\
\hline (a) & 1.0 & 9954 & 25300 & 6433.45 & $\mathrm{Q} \uparrow \mathrm{Z} \downarrow$ \\
(b) & 2.5 & 9785 & 25399 & 6452.40 & $\mathrm{Q} \uparrow \mathrm{Z} \downarrow$ \\
Basic case & 5.0 & 9485 & 25443 & 6465.32 & - \\
(c) & 7.5 & 9268 & 25605 & 6508.85 & $\mathrm{Q} \downarrow \mathrm{Z} \uparrow$ \\
(d) & 10 & 8862 & 25647 & 6542.35 & $\mathrm{Q} \downarrow \mathrm{Z} \uparrow$ \\
\hline
\end{tabular}

\section{Conclusion}

In the literature, most existing EPQ models for imperfect processes do not consider statistical quality control methods such as acceptance sampling plans, although, these methods are widely employed in industry. The hypothesis of $100 \%$ inspection of all produced items is far from being used in practice because of its high cost. In this paper, we addressed the problem of joint determination of the optimal lot sizing and production-inventory control policy for unreliable and imperfect manufacturing systems where quality control is performed using a single acceptance sampling plan. The problem was formulated with a stochastic dynamic programming model in which the lot sizing and the production rate are considered as decision variables. Due to the high stochastic level of the model and the complexity of the system dynamics, it was shown that the optimization problem is intractable either analytically or numerically. However, we proposed a heuristic feedback control policy based on a combination of a modified HPP and batch processing control policy. The efficiency of the control policy was illustrated using a simulation-based experimental approach. The main advantage of this approach is that provides the possibility of

establishing a realistic representation of the stochastic and dynamic behaviour of the system using a combined discrete-continuous simulation model, and optimizing the control policy parameters. A thorough sensitivity analysis was performed and some interesting behaviours were observed, which underscores the strong and complex interaction between the production and quality aspects in real manufacturing systems. Possible extensions of this work can be envisaged when quality control is performed using double or multiple acceptance sampling plans. As well, further research could be carried out to investigate the joint optimization of the production control policy and sampling plan parameters.

\section{References}

Akella, R., \& Kumar, P. (1986). Optimal control of production rate in a failure prone manufacturing system. IEEE Transactions on Automatic Control, 31(2), 116-126.

Ben-Daya, M. (1999). Integrated production maintenance and quality model for imperfect processes. IIE Transactions, 31(6), 491-501.

Ben-Daya, M. (2002). The economic production lot-sizing problem with imperfect production processes and imperfect maintenance. International Journal of Production Economics, 76(3), 257-264. 
Ben-Daya, M., Noman, S., \& Hariga, M. (2006). Integrated inventory control and inspection policies with deterministic demand. Computers \& Operations Research, 33(6), 1625-1638.

Ben-Daya, M., \& Noman, S. (2008). Integrated inventory and inspection policies for stochastic demand. European Journal of Operational Research, 185(1), 159-169.

Besterfield, D. H. (2009). Quality Control: Prentice Hall.

Bielecki, T., \& Kumar, P. (1988). Optimality of zero-inventory policies for unreliable manufacturing systems. Operations Research, 36(4), 532-541.

Boukas, E. K., \& Haurie, A. (1990). Manufacturing flow control and preventing maintenance: a stochastic control approach. IEEE Transactions on Automatic Control, 35(9), 1024-1031.

Boukas, E., Shi, P., \& Agarwal, R. (2000). An application of robust control technique to manufacturing systems with uncertain processing time. Optimal Control Applications and Methods, 21(6), 257-268.

Bouslah, B., Gharbi, A., Pellerin, R., and Hajji, A. (2012). Optimal production control policy in unreliable batch processing manufacturing systems with transportation delay. International Journal of Production Research, In press. DOI:10.1080/00207543.2012.676217.

Chakraborty, T., Giri, B., \& Chaudhuri, K. (2009). Production lot sizing with process deterioration and machine breakdown under inspection schedule. Omega, 37(2), 257-271.

Chin, R. T., \& Harlow, C. A. (1982). Automated visual inspection: A survey. IEEE Transactions on Pattern Analysis and Machine Intelligence, (6), 557-573.

Chiu, P. (2003). Determining the optimal lot size for the finite production model with random defective rate, the rework process, and backlogging. Engineering Optimization, 35(4), 427-437.

Chiu, S. W., Wang, S. L., \& Chiu, Y. S. P. (2007). Determining the optimal run time for EPQ model with scrap, rework, and stochastic breakdowns. European Journal of Operational Research, 180(2), 664-676.

Chiu, W. (1974). A new prior distribution for attributes sampling. Technometrics, 16(1), 93-102.

Chung, K. J. (1997). Bounds for production lot sizing with machine breakdowns. Computers \& Industrial Engineering, 32(1), 139-144.

Gershwin, S. B. (1994). Manufacturing Systems Engineering: Prentice Hall.

Gharbi, A., \& Kenne, J. (2000). Production and preventive maintenance rates control for a manufacturing system: an experimental design approach. International Journal of Production Economics, 65(3), 275-287.

Gharbi, A., \& Kenne, J. P. (2003). Optimal production control problem in stochastic multipleproduct multiple-machine manufacturing systems. IIE Transactions, 35(10), 941-952.

Giri, B., \& Dohi, T. (2005). Computational aspects of an extended EMQ model with variable production rate. Computers \& Operations Research, 32(12), 3143-3161.

Giri, B., Yun, W., \& Dohi, T. (2005). Optimal design of unreliable production-inventory systems with variable production rate. European Journal of Operational Research, 162(2), 372386. 
Groenevelt, H., Pintelon, L., \& Seidmann, A. (1992a). Production lot sizing with machine breakdowns. Management Science, 38(1), 104-123.

Groenevelt, H., Pintelon, L., \& Seidmann, A. (1992b). Production batching with machine breakdowns and safety stocks. Operations Research, 40(5), 959-971.

Hald, A. (1960). The compound hypergeometric distribution and a system of single sampling inspection plans based on prior distributions and costs. Technometrics, 2(3), 275-340.

Hayek, P. A., \& Salameh, M. K. (2001). Production lot sizing with the reworking of imperfect quality items produced. Production Planning \& Control, 12(6), 584-590.

Hu, J. Q., Vakili, P., \& Huang, L. (2004). Capacity and Production Managment in a single Product Manufacturing system. Annals of Operations Research, 125(1), 191-204.

Khan, M., Jaber, M. Y., Guiffrida, A. L., \& Zolfaghari, S. (2011). A review of the extensions of a modified EOQ model for imperfect quality items. International Journal of Production Economics, 132(1), 1-12.

Khouja, M., \& Mehrez, A. (1994). Economic production lot size model with variable production rate and imperfect quality. The Journal of the Operational Research Society, 45(12), 14051417.

Kim, C. H., \& Hong, Y. (1997). An extended EMQ model for a failure prone machine with general lifetime distribution. International Journal of Production Economics, 49(3), 215223.

Kim, C. H., Hong, Y., \& Kim, S.-Y. (1997). An extended optimal lot sizing model with an unreliable machine. Production Planning \& Control: The Management of Operations, $8(6), 577-585$.

Lavoie, P., Kenné, J. P., \& Gharbi, A. (2007). Production control and combined discrete/continuous simulation modeling in failure-prone transfer lines. International Journal of Production Research, 45(24), 5667-5685.

Lavoie, P., Gharbi, A., \& Kenné, J. P. (2010). A comparative study of pull control mechanisms for unreliable homogenous transfer lines. International Journal of Production Economics, 124(1), 241-251.

Law, A.M., \& David, W.D. (2000). Simulation modeling and analysis: McGraw-Hill.

Lee, H. L., \& Rosenblatt, M. J. (1987). Simultaneous determination of production cycle and inspection schedules in a production systems. Management Science, 1125-1136.

Li, J., Sava, A., \& Xie, X. (2009). An analytical approach for performance evaluation and optimization of a two-stage production-distribution system. International Journal of Production Research, 47(2), 403-414.

Liao, G. L., Chen, Y. H., \& Sheu, S. H. (2009). Optimal economic production quantity policy for imperfect process with imperfect repair and maintenance. European Journal of Operational Research, 195(2), 348-357.

Min, H., \& Galle, W. P. (1997). Green purchasing strategies: trends and implications. Journal of Supply Chain Management, 33(3), 10-17.

Montgomery, D. C. (2008). Design and analysis of experiments: John Wiley \& Sons Inc. 
Mourani, I., Hennequin, S., \& Xie, X. (2008). Simulation-based optimization of a single-stage failure-prone manufacturing system with transportation delay. International Journal of Production Economics, 112(1), 26-36.

Myers, R. H., Montgomery, D. C., \& Anderson-Cook, C. M. (2009). Response surface methodology: process and product optimization using designed experiments (Vol. 705): Wiley.

Noguera, J. H., \& Watson, E. F. (2006). Response surface analysis of a multi-product batch processing facility using a simulation metamodel. International Journal of Production Economics, 102(2), 333-343.

Pegden, C. D., Shannon, R. E., Sadowski, R. P., \& Corp, S. M. (1995). Introduction to simulation using SIMAN: McGraw-Hill.

Porteus, E. L. (1986). Optimal lot sizing, process quality improvement and setup cost reduction. Operations Research, 137-144.

Rosenblatt, M. J., \& Lee, H. L. (1986). Economic production cycles with imperfect production processes. IIE Transactions, 18(1), 48-55.

Salameh, M., \& Jaber, M. (2000). Economic production quantity model for items with imperfect quality. International Journal of Production Economics, 64(1-3), 59-64.

Sana, S. S. (2010). An economic production lot size model in an imperfect production system. European Journal of Operational Research, 201(1), 158-170.

Sana, S. S., \& Chaudhuri, K. (2010). An EMQ model in an imperfect production process. International Journal of Systems Science, 41(6), 635-646.

Schilling, E. G., \& Neubauer, D. V. (2009). Acceptance sampling in quality control: Chapman \& Hall/CRC.

Stephens, K. S. (2001). Handbook of Applied Acceptance Sampling: Plans, Procedures \& Principles: ASQ Quality Press Milwaukee, WI. 